\title{
A FINITE ELEMENT METHOD FOR TIME-DEPENDENT CONVECTION-DIFFUSION EQUATIONS
}

\author{
GERARD R. RICHTER
}

\begin{abstract}
We present a finite element method for time-dependent convectiondiffusion equations. The method is explicit and is applicable with piecewise polynomials of degree $n \geq 2$. In the limit of zero diffusion, it reduces to a recently analyzed finite element method for hyperbolic equations. Near optimal error estimates are derived. Numerical results are given.
\end{abstract}

\section{INTRODUCTION}

In this paper we describe and analyze a new finite element method for timedependent convection-diffusion equations. The method employs space-time elements of arbitrarily high order and is "explicit", allowing the approximate solution to be computed one element at a time. It is tailored to the hyperbolic limit and is potentially well suited to convection-dominated problems.

Our framework will be the model equation

$$
u_{\alpha}=\sigma u_{x x}+f(x, t), \quad(x, t) \in \Omega,
$$

where $\Omega$ is a rectangular domain in $R^{2}$. The term $u_{\alpha}$ denotes the directional derivative $\alpha \cdot \nabla u=\alpha_{1} u_{x}+\alpha_{2} u_{t}$, where $\alpha$ is a unit vector with $\alpha_{2}>0$. We assume $\alpha$ and $\sigma$ are constant, and that appropriate Dirichlet data is prescribed on the boundary $\Gamma$ of $\Omega$.

The basis of our method is the hyperbolic limit of (1.1):

$$
u_{\alpha}=f \quad \text { in } \Omega \text {. }
$$

For (1.2) we assume $u$ is given on the inflow portion of $\Gamma$,

$$
\Gamma_{\text {in }}(\Omega) \equiv\{(x, t) \in \Gamma \mid \alpha \cdot n<0\},
$$

where $n$ is the unit outer normal to $\Omega$. Given a triangulation of $\Omega$, one may develop a finite element approximation for (1.2) in an explicit manner, processing the triangles one at a time in an order consistent with domain of dependence requirements. That such an ordering always exists is shown in [4]. Our finite element approximation $u_{h}$ will be a continuous piecewise polynomial of degree $\leq n$. We start $u_{h}$ as a standard interpolant (e.g., piecewise equinodal) on $\bar{\Gamma}_{\text {in }}(\Omega)$, and define it in individual triangles $T$ by the conditions

$$
\left(\left(u_{h}\right)_{\alpha}, v_{h}\right)_{T}=\left(f, v_{h}\right)_{T}, \quad \text { all } v_{h} \in \mathbf{P}_{n-\rho(T)}(T) .
$$

Received July 12, 1988; revised February 23, 1989.

1980 Mathematics Subject (Inssification (1985 Revision). Primary 65N30, 65M15.

Key' words and phrase's. Finite element, hyperbolic. 
Here, $(,)_{T}$ is the $L^{2}(T)$ inner product, $\mathbf{P}_{k}(T)$ is the space of polynomials of degree $\leq k$ over $T$, and $\rho(T)$ is the number of inflow sides that $T$ has. Assuming no triangle side is parallel to $\alpha, \rho(T)$ is unequivocally equal to either 1 (a type I triangle) or 2 (a type II triangle). For a triangle $T$ of either type, the dimension of the test space in (1.3) is equal to the number of remaining degrees of freedom for $u_{h}$ in $T$, given that it will already be known on the inflow side(s) of $T$ when conditions (1.3) are imposed. We assume $n \geq 2$, for if $n=1$ the inner product conditions (1.3) are vacuous for a type II triangle.

The method (1.3) was proposed by Reed and Hill [6]. In [1], the approximate solution was shown to satisfy an error bound of the form

$$
\left\|u_{h}-u\right\|_{L^{2}(\Omega)} \leq C h^{n+1 / 4}\|u\|_{H^{n+1}(\Omega)},
$$

although the optimal $O\left(h^{n+1}\right)$ convergence rate is commonly observed computationally. Numerical evidence [1] also indicates that the method handles discontinuities well, with relatively little spurious propagation to other parts of the domain. These properties, together with the fact that it employs a continuous approximation subspace, make the method a potentially attractive candidate for extension to convection-dominated convection-diffusion problems.

Returning to the convection-diffusion problem (1.1), we retain the distinction between type I and type II triangles, based on the convection term. One way to accommodate the presence of diffusion would be to simply include an additional inner product of $\sigma\left(u_{h}\right)_{x x}$ against $v_{h}$ in (1.3). However, we have found that the following alternative yields better accuracy for convection-dominated problems:

$$
\begin{gathered}
\left(\left(u_{h}\right)_{\alpha}-\sigma\left(u_{h}\right)_{x x}, v_{h}\right)_{T}+\sigma \int_{\Gamma_{\text {in }}^{*}(T)}\left[\left(u_{h}\right)_{x}^{+}-\left(u_{h}\right)_{x}^{-}\right] v_{h} e_{1} \cdot n d \tau \\
=\left(f, v_{h}\right)_{T}, \quad \text { all } v_{h} \in \mathbf{P}_{n-\rho(T)}(T) .
\end{gathered}
$$

Here, $\Gamma_{\text {in }}^{*}(T)$ is the inflow boundary of $T$, exclusive of any sides lying on the boundary of $\Omega$, if $T$ should have any. For a point $P \in \Gamma_{\text {in }}^{*}(T)$, where the first derivative of $u_{h}$ is in general discontinuous,

$$
\left(u_{h}\right)_{x}^{ \pm}(P) \equiv \lim _{\varepsilon \rightarrow 0+}\left(u_{h}\right)_{x}(P \pm \varepsilon \alpha)
$$

The integral over $\Gamma_{\text {in }}^{*}(T)$ is taken with respect to arclength, with $e_{1}$ and $n$ denoting unit vectors in the $x$ and outer normal directions (equivalently, $e_{1} \cdot n=n_{1}$, the horizontal component of $\left.n\right)$. The $\Gamma_{\text {in }}^{*}(T)$ integral in (1.5) produces a more tractable boundary term when (1.5) is integrated by parts. An analogous boundary integral appears in the discontinuous Galerkin method for (1.2) [3].

The finite element method (1.5) is explicit. Accordingly, a restriction on the size of

$$
q \equiv \frac{\sigma k}{h^{2}}
$$

emerges as a stability condition, where $h$ and $k$ are the triangle projections onto the $x$ and $t$ axes. In a convection-dominated case with $\sigma \leq h$, this need not be a severe restriction. Moreover, the "time step" $k$ may be varied spatially, 
allowing development of the approximation in a time-staggered manner rather than along lines $t=$ constant. This added flexibility could be advantageous in a problem where the maximum stable time step exhibits a strong spatial dependence.

Assuming the stability condition is met, we will establish error estimates of the form

$$
\begin{gathered}
\left\|u_{h}-u\right\|_{L^{2}(\Omega)} \leq C q^{-1} \sqrt{\sigma} h^{n}\|u\|_{H^{n+1}(\Omega)}, \\
\left\|\left(u_{h}-u\right)_{X}\right\|_{L^{2}(\Omega)} \leq C q^{-1} h^{n}\|u\|_{H^{n+1}(\Omega)},
\end{gathered}
$$

where $C$ signifies a generic constant, independent of $u$ and the triangulation. For fixed $\sigma>0,(1.7)$ and (1.8) give $O\left(h^{n}\right)$ error bounds for both $u_{h}$ and $\left(u_{h}\right)_{x}$. For a family of problems in which $\sigma$ is proportional to $h$, the bound on $u_{h}$ improves to $O\left(h^{n+1 / 2}\right)$. This is the same as that obtained in [2,5] for the streamline diffusion method with $\sigma \leq h$. The latter method requires $\Omega$ to be triangulated in strips $t_{i} \leq t \leq t_{i+1}$, and the approximation is implicit within the strips. We note that the bounds (1.7) and (1.8) become meaningless as $\sigma \rightarrow 0$ for a fixed triangulation, for in this limit, $q \rightarrow 0$ too. A refinement of the basic analysis will handle this limit correctly, producing in place of $(1.7),(1.8)$ the corresponding hyperbolic estimates, $O\left(h^{n+1 / 4}\right), O\left(h^{n-1 / 2}\right)$, given in [1].

In the following four sections, we analyze the finite element scheme under the assumption that $(1.1)$ and its discretization are spatially periodic. This precludes the possibility of physical inflow or outflow boundaries. In $\S 2$ we state our assumptions on the triangulation and show that the approximate solution is well defined provided $q$ is sufficiently small. In $\S 3$ we obtain a global stability result and then use it in $\S 4$ to obtain the error estimates (1.7) and (1.8). The hyperbolic limit $\sigma \rightarrow 0$ is dealt with in $\S 5$. In $\S 6$ we remove the assumption of periodicity in $x$. The prescription (1.5) must then be altered for triangles with a side lying along the outflow boundary where "parabolic" boundary data for $u$ is given. In the convection-dominated case $\sigma \leq O(h)$, the resulting disturbance can be confined to an outflow boundary layer. Computational results are given in $\S 7$.

\section{Preliminaries}

Here we state our basic assumptions and prove existence and uniqueness of the approximate solution.

Our analysis will apply to a family of problems (1.1) for which $\alpha_{2}$ is uniformly positive and $\sigma>0$. In $\S \S 2-5$, we assume (1.1) and its discretization are spatially periodic and take $\Omega$ to consist of a single period, $[0, X] \times(0, T)$, say. This allows us to defer the issue of spatial boundary conditions to a later section, simplifying the exposition. Figure 7.1 a illustrates such a situation, with $u_{h}$ evolving (explicitly) upward through alternate layers of type I and type II triangles. In addition to being periodic, we assume our triangulations have the following properties:

H1. The ratio of the maximum to minimum $h$ over a triangulation is uniformly bounded, and similarly for $k$. 
H2. There exists a constant $\kappa_{1}>0$ such that

$$
\sin \theta \geq \kappa_{1} \frac{k}{h}
$$

for each interior angle $\theta$ of each triangle.

H3. There exists a constant $\kappa_{2}>0$ such that for all triangle sides

$$
\left|e_{1} \cdot n\right| \leq \kappa_{2} \frac{k}{h} .
$$

H4. $|\alpha \cdot n|$ is uniformly bounded away from zero over all sides of all triangles.

Assumptions $\mathrm{H} 1$ and $\mathrm{H} 2$ imply that our triangulations are the result of compressing by factors $h, k$ in directions $x, t$, respectively, a set of quasi-uniform triangulations with side length $O(1)$ and minimum angle bounded away from zero. H3 will be needed to permit upstream propagation of "parabolic" information, and $\mathrm{H} 4$ is a requirement of the hyperbolic analysis in [1]. Conditions $\mathrm{H} 1-\mathrm{H} 3$ are automatically satisfied by a family of uniform isosceles triangulations like that shown in Figure 7.1a, provided $k \leq O(h)$. Note that, in general, $\mathrm{H} 2$ implies that $k \leq O(h)$ as $h \rightarrow 0$.

For a generic triangle $T$, we denote by \|\|$_{T}$ and ||$_{\Gamma(T)}$ the interior and boundary $L^{2}$ norms. The following bounds are easily obtained:

Lemma 2.1. For $v_{h} \in \mathbf{P}_{n}(T)$,

$$
\begin{aligned}
& \text { (i) }\left|v_{h}\right|_{\Gamma(T)} \leq C \frac{\left\|v_{h}\right\|_{T}}{\sqrt{k}} \\
& \text { (ii) }\left\|\left(v_{h}\right)_{x}\right\|_{T} \leq C h^{-1}\left\|v_{h}\right\|_{T} \\
& \text { (iii) }\left\|\left(v_{h}\right)_{\alpha}\right\|_{T} \leq C k^{-1}\left\|v_{h}\right\|_{T} \\
& \text { (iv) If } v_{h}=0 \text { at some point of } T, \\
& \\
& \quad\left\|v_{h}\right\|_{T} \leq C\left(k\left\|\left(v_{h}\right)_{\alpha}\right\|_{T}+h\left\|\left(v_{h}\right)_{x}\right\|_{T}\right) .
\end{aligned}
$$

We now establish existence and uniqueness of the approximate solution. For a triangle $T$ with dimensions $h$ and $k$, we define

$$
\hat{x}=\frac{x}{h}, \quad \hat{t}=\frac{t}{k},
$$

and transform the inner product relations (1.5) to a "reference" triangle $\hat{T}$ for which $\hat{h}=\hat{k}=1$. The result can be written

$$
\begin{aligned}
& \left(\left(\hat{u}_{h}\right)_{\hat{x}}-\hat{\sigma}\left(\hat{u}_{h}\right)_{\hat{x} \hat{x}}, \hat{v}_{h}\right)_{\hat{T}}+\hat{\sigma} \int_{\Gamma_{\text {in }}^{*}(\hat{T})}\left(\hat{u}_{h}\right)_{\hat{x}}^{+} \hat{v}_{h} \hat{e}_{1} \cdot \hat{n} d \hat{\tau} \\
& \quad=\hat{\beta}\left(\hat{f}, \hat{v}_{h}\right)_{\hat{T}}+\hat{\sigma} \int_{\Gamma_{\text {in }}^{*}(\hat{T})}\left(\hat{u}_{h}\right)_{\hat{x}}^{-} \hat{v}_{h} \hat{e}_{1} \cdot \hat{n} d \hat{\tau}, \quad \text { all } \hat{v}_{h} \in \mathbf{P}_{n-\rho(\hat{T})}(\hat{T}),
\end{aligned}
$$


where

$$
\begin{aligned}
& \hat{u}_{h}(\hat{x}, \hat{t})=u_{h}(x, t), \quad \hat{f}(\hat{x}, \hat{t})=f(x, t), \\
& \hat{\alpha}=\frac{1}{\sqrt{1+\left(\frac{\alpha_{1} k}{\alpha_{2} h}\right)^{2}}}\left(\begin{array}{c}
\frac{\alpha_{1} k}{\alpha_{2} h} \\
1
\end{array}\right), \\
& \hat{\sigma}=\frac{\frac{q}{\alpha_{2}}}{\sqrt{1+\left(\frac{\alpha_{1} k}{\alpha_{2} h}\right)^{2}}},
\end{aligned}
$$$$
\hat{\beta}=\frac{\frac{k}{\alpha_{2}}}{\sqrt{1+\left(\frac{\alpha_{1} k}{\alpha_{2} h}\right)^{2}}} .
$$

Note that (2.7) is to be supplemented by given values for $\hat{u}_{h}$ on $\Gamma_{\text {in }}(\hat{T})$.

Representing $\hat{u}_{h}$ in Lagrange form in terms of its values $\hat{u}$ at the standard equispaced nodes for $n$th degree interpolation, then applying the inflow conditions and enforcing (2.7) for the Lagrange basis functions for $\mathbf{P}_{n-\rho(\hat{T})}(\hat{T})$, we obtain a linear algebraic system

$$
A \hat{u}=b \text {, }
$$

where

$$
\max _{i}\left|b_{i}\right| \leq C\left(\left|\hat{u}_{h}\right|_{\Gamma_{\mathrm{in}}(\hat{T})}+\hat{\sigma}\left|\left(\hat{u}_{h}\right)_{\hat{x}}\right|_{\Gamma_{\mathrm{in}}^{*}(\hat{T})}+\hat{\beta}\|\hat{f}\|_{\hat{T}}\right)
$$

To establish uniqueness (hence existence), we show that if $\hat{f} \equiv 0$ in $\hat{T}, \hat{u}_{h}=0$ on $\Gamma_{\text {in }}(\hat{T})$, and $\left(\hat{u}_{h}\right)_{\hat{x}}^{-}=0$ on $\Gamma_{\text {in }}^{*}(\hat{T})$ (making $b=0$ in $(2.10)$ ), then $\hat{u}=0$ also, assuming $\hat{\sigma}$ is sufficiently small.

Lemma 2.2. There exists a constant $\hat{\sigma}_{0}>0$ such that for $\sigma \in\left[0, \hat{\sigma}_{0}\right]$, the only solution of

$$
\begin{aligned}
& \left(\left(\hat{u}_{h}\right)_{\hat{\alpha}}-\hat{\sigma}\left(\hat{u}_{h}\right)_{\hat{x} \hat{x}}, \hat{v}_{h}\right)_{\hat{T}}+\hat{\sigma} \int_{\Gamma_{\text {in }}^{*}(\hat{T})}\left(\hat{u}_{h}\right)_{\hat{x}}^{+} \hat{v}_{h} \hat{e}_{1} \cdot \hat{n} d \hat{\tau}=0, \\
& \text { all } \hat{v}_{h} \in \mathbf{P}_{n-\rho(\hat{T})}(\hat{T}), \\
& \hat{u}_{h}=0 \text { on } \Gamma_{\text {in }}(\hat{T})
\end{aligned}
$$

is $\hat{u}_{h} \equiv 0$ in $\hat{T}$.

Proof. Since $\hat{u}_{h}=0$ on $\Gamma_{\text {in }}(\hat{T})$, we may factor from $\hat{u}_{h}$ a function $\hat{\zeta} \epsilon$ $\mathbf{P}_{\rho(\hat{T})}(\hat{T})$ for which $\hat{\zeta}=0$ on $\Gamma_{\text {in }}(\hat{T})$. An illustration appears in Figure 2.1. For a type I triangle, $\hat{\zeta}$ is the distance from the inflow side; for a type II triangle, $\hat{\zeta}$ is the product of coordinates $\hat{\xi}$ and $\hat{\eta}$ aligned with the two inflow sides. 


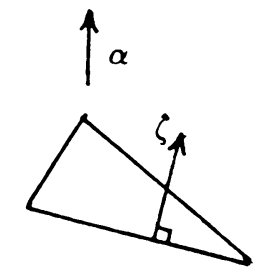

Type I triangle

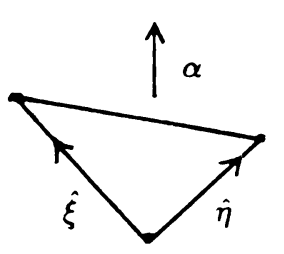

Type II triangle

FIGURE 2.1

We thus have the representation

$$
\hat{u}_{h}=\hat{\zeta}_{h}, \quad \hat{\zeta}=0 \text { on } \Gamma_{\text {in }}^{*}(\hat{T}), \quad \hat{w}_{h} \in \mathbf{P}_{n-\rho(\hat{T})}(\hat{T}) .
$$

We choose $\hat{v}_{h}$ in (2.12) to be $\hat{w}_{h}$. Integrating twice by parts and using the fact that $(\hat{\zeta})_{\dot{r}}>0$ in the interior of $\hat{T}$, we obtain

$$
\begin{aligned}
\left(\left(\hat{u}_{h}\right)_{\dot{i}}, \hat{w}_{h}\right)_{\hat{T}} & =\left(\left(\hat{\zeta} \hat{w}_{h}\right)_{\dot{i}}, \hat{w}_{h}\right)_{\hat{T}} \\
& =-\frac{1}{2}\left(\hat{\zeta},\left(\hat{w}_{h}^{2}\right)_{\dot{\hat{r}}}\right)_{\hat{T}}+\int_{\Gamma_{\text {oul }}(\hat{T})} \hat{\zeta} \hat{w}_{h}^{2} \hat{\alpha} \cdot \hat{n} d \hat{\tau} \\
& =\frac{1}{2}\left((\hat{\zeta})_{\dot{i}}, \hat{w}_{h}^{2}\right)_{\hat{T}}+\frac{1}{2} \int_{\Gamma_{\text {out }}(\hat{T})} \hat{\zeta} \hat{w}_{h}^{2} \hat{\alpha} \cdot \hat{n} d \hat{\tau} \\
& \geq \frac{1}{2}\left((\hat{\zeta})_{\dot{i}}, \hat{w}_{h}^{2}\right)_{\hat{T}} \geq \mu\left\|\hat{w}_{h}^{2}\right\|_{\hat{T}}^{2},
\end{aligned}
$$

where $\mu$ is a positive constant. Using inverse inequalities ard equivalence of norms in the reference triangle,

$$
\begin{aligned}
& -\hat{\sigma}\left(\left(\hat{u}_{h}\right)_{\hat{x} \hat{r}}, \hat{w}_{h}\right)_{\hat{T}}+\hat{\sigma} \int_{\Gamma_{\text {in }}^{*}(\hat{T})}\left(\hat{u}_{h}\right)_{\hat{x}}^{+} \hat{w}_{h} \hat{e}_{1} \cdot \hat{n} d \hat{\tau} \\
& \quad \leq \hat{\sigma}\left\|\left(\hat{\zeta} \hat{w}_{h}\right)_{\hat{x} \hat{r}}\right\|_{\dot{T}} \cdot\left\|\hat{w}_{h}\right\|+\hat{\sigma}\left|\left(\hat{\zeta} \hat{w}_{h}\right)_{\hat{x}}\right|_{\Gamma_{\text {in }}(\hat{T})} \cdot\left|\hat{w}_{h}\right|_{\Gamma_{\text {in }}(\hat{T})} \\
& \quad \leq C \hat{\sigma}\left\|\hat{\zeta} \hat{w}_{h}\right\|_{\hat{T}} \cdot\left\|\hat{w}_{h}\right\|_{\hat{T}} \leq C \hat{\sigma}\left\|\hat{w}_{h}\right\|_{\hat{T}}^{2} .
\end{aligned}
$$

Thus the choice $\hat{v}_{h}=\hat{w}_{h}$ in (2.12) gives

$$
\mu\left\|\hat{w}_{h}\right\|_{\hat{T}}^{2}-C \hat{\sigma}\left\|\hat{w}_{h}\right\|_{\hat{T}}^{2} \leq 0 .
$$

This implies $\hat{w}_{h} \equiv 0$ (hence $\hat{u}_{h} \equiv 0$ ) for sufficiently small $\hat{\sigma}$, e.g., for $\hat{\sigma} \in$ $\left[0, \frac{\mu}{2 C}\right]$.

The parameters on which $A$ in (2.10) depends are the reference triangle $\hat{T}$, unit vector $\hat{\alpha}$, and $\hat{\sigma} \in\left[0, \hat{\sigma}_{0}\right]$. The set $S$ of all such parameters is closed and bounded, and $\left\|A^{-1}\right\|_{\infty}$ is continuous over it. Therefore, $\sup _{S}\left\|A^{-1}\right\|_{\infty}$ is attained at an element of $S$, implying the existence of a uniform bound on $\left\|A^{-1}\right\|_{\infty}$. Hence, from (2.11),

$$
\left\|\hat{u}_{h}\right\|_{\hat{T}} \leq C\left(\left|\hat{u}_{h}\right|_{\Gamma_{\text {in }}(\hat{T})}+\hat{\sigma}\left|\left(\hat{u}_{h}\right)_{\hat{x}}^{-}\right|_{\Gamma_{\text {in }}^{*}(\hat{T})}+\beta\|\hat{f}\|_{\hat{T}}\right) .
$$


Transforming back to the actual triangle $T$, applying (2.8) and (2.9), and recalling that $k \leq O(h)$, we obtain

Lemma 2.3. For $q$ sufficiently small,

$$
\left\|u_{h}\right\|_{T} \leq C\left(\sqrt{k}\left|u_{h}\right|_{\Gamma_{\text {in }}(T)}+q h \sqrt{k}\left|\left(u_{h}\right)_{x}^{-}\right|_{\Gamma_{\text {in }}^{*}(T)}+k\|f\|_{T}\right) .
$$

\section{Stability}

In this section we derive a global stability result for the finite element scheme, implicitly assuming $q$ to be small enough so that $u_{h}$ is well defined. A key test function of the hyperbolic analysis [1] was $\left(u_{h}\right)_{\tau_{1} \tau_{2}}$, where $\tau_{1}$ and $\tau_{2}$ denote the unit tangents to the two outflow (inflow) sides to a type I (type II) triangle, taken counterclockwise, and $\left(u_{h}\right)_{\tau_{1} \tau_{2}}$ is the corresponding mixed derivative. (See Figure 3.1.) Fortunately, this is also a good test function for the diffusion term $\sigma\left(u_{h}\right)_{x x}$. For if $\sigma$ is a positive constant, $k=O\left(h^{2}\right)$ is required for boundedness of $q$. Thus by assumption $\mathrm{H} 3$, triangle sides must become more nearly horizontal as $h \rightarrow 0$, and this causes $\left(u_{h}\right)_{\tau_{1} \tau_{2}}$ to behave like $\left(u_{h}\right)_{x x}$. Another key test function which works well for both convection and diffusion terms is $P_{n-2} u_{h}$, where $P_{n-2}$ is the $L^{2}$ projection operator into $\mathbf{P}_{n-2}(T)$. Together, these test functions lead to control over $u_{h}$ and its derivatives.

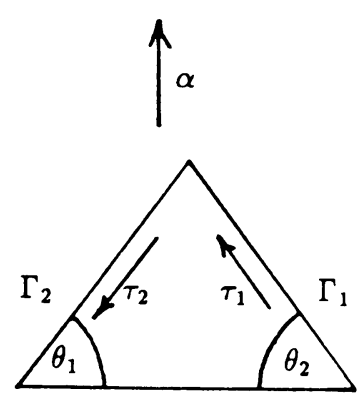

$\Gamma_{3}$

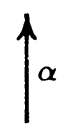

$\Gamma_{3}$

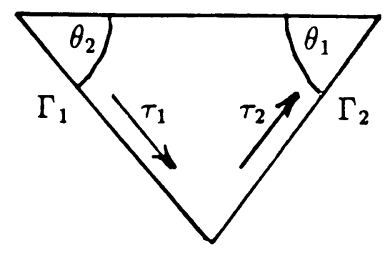

Type II triangle

FIGURE 3.1

In what follows, all norms and inner products are taken over $T$ unless otherwise signified, and $\oint(\cdot)$ will denote an integral around the boundary $\Gamma(T)$. We denote by $I(T)$ the triangle(s) lying along the upstream side of $\Gamma_{\text {in }}^{*}(T)$; if $\Gamma_{\text {in }}^{*}(T)=\varnothing$ we define $I(T)=\varnothing$ also.

We first bound the inflow boundary integral in (1.5) in terms of $\left(u_{h}\right)_{n}$, a quantity that can be controlled directly.

Lemma 3.1. The integral over $\Gamma_{\text {in }}^{*}(T)$ in (1.5) can be bounded as follows:

$$
\left|\sigma \int_{\Gamma_{\text {in }}^{*}(T)}\left[\left(u_{h}\right)_{x}^{+}-\left(u_{h}\right)_{x}^{-}\right] v_{h} e_{1} \cdot n d \tau\right| \leq C q\left\|\left(u_{h}\right)_{r}\right\|_{T \cup I(T)} \cdot\left\|v_{h}\right\| .
$$


Proof. We assume $\Gamma_{\text {in }}^{*}(T) \neq \varnothing$, for otherwise the result is trivially true. Via the Schwarz inequality, (2.2), and (2.3),

$$
\left|\sigma \int_{\Gamma_{\text {in }}^{*}(T)}\left[\left(u_{h}\right)_{x}^{+}-\left(u_{h}\right)_{x}^{-}\right] v_{h} e_{1} \cdot n d \tau\right| \leq C \sigma \frac{\sqrt{k}}{h}\left|\left(u_{h}\right)_{x}^{+}-\left(u_{h}\right)_{x}^{-}\right|_{\Gamma_{\text {in }}^{*}(T)} \cdot\left\|v_{h}\right\| .
$$

Let $\tau$ denote the unit tangent along the boundary of $T$, measured clockwise, and $n=\left(\begin{array}{l}n_{1} \\ n_{2}\end{array}\right)$ the unit outer normal. The directional derivatives $\left(u_{h}\right)_{\alpha}$ and $\left(u_{h}\right)_{\tau}$ along $\Gamma_{\text {in }}^{*}(T)$ are given by

$$
\left(\begin{array}{l}
\left(u_{h}\right)_{\alpha} \\
\left(u_{h}\right)_{\tau}
\end{array}\right)=\left(\begin{array}{cc}
\alpha_{1} & \alpha_{2} \\
-n_{2} & n_{1}
\end{array}\right)\left(\begin{array}{c}
\left(u_{h}\right)_{x} \\
\left(u_{h}\right)_{t}
\end{array}\right)
$$

whose inverse is

$$
\left(\begin{array}{c}
\left(u_{h}\right)_{x} \\
\left(u_{h}\right)_{t}
\end{array}\right)=\frac{1}{\alpha \cdot n}\left(\begin{array}{cc}
n_{1} & -\alpha_{2} \\
n_{2} & \alpha_{1}
\end{array}\right)\left(\begin{array}{l}
\left(u_{h}\right)_{\alpha} \\
\left(u_{h}\right)_{\tau}
\end{array}\right)
$$

Since $\left(u_{h}\right)_{\tau}$ is continuous along $\Gamma_{\text {in }}^{*}(T)$,

$$
\left(u_{h}\right)_{x}^{+}-\left(u_{h}\right)_{x}^{-}=\frac{n_{1}}{\alpha \cdot n}\left[\left(u_{h}\right)_{\alpha}^{+}-\left(u_{h}\right)_{\alpha}^{-}\right] \text {. }
$$

Thus by assumption $\mathrm{H} 3, \mathrm{H} 4$, and (2.3),

$$
\left|\left(u_{h}\right)_{x}^{+}-\left(u_{h}\right)_{x}^{-}\right|_{\Gamma_{\text {in }}^{*}(T)} \leq C \frac{k}{h}\left|\left(u_{h}\right)_{\alpha}^{+}-\left(u_{h}\right)_{\alpha}^{-}\right|_{\Gamma_{\text {in }}^{*}(T)} \leq C-\frac{\sqrt{k}}{h}\left\|\left(u_{h}\right)_{\alpha}\right\|_{T \cup I(T)} .
$$

Insertion into (3.2) gives the desired result.

We now show:

Lemma 3.2. There exists a constant $\xi>0$ such that

$$
\begin{gathered}
\oint u_{h}^{2} \alpha \cdot n d \tau+\xi \sigma\left\|\left(u_{h}\right)_{x}\right\|^{2}-2 \sigma \int_{\Gamma^{*}(T)}\left(u_{h}\right)_{x}^{-} u_{h} e_{1} \cdot n d \tau \\
\leq 2\left(f, u_{h}\right)+C \frac{k}{q}\left(\left\|\left(u_{h}\right)_{\alpha}\right\|_{T \cup I(T)}^{2}+\|f\|^{2}\right),
\end{gathered}
$$

where $\Gamma^{*}(T)=\Gamma(T)-\left(\Gamma(T) \cap \Gamma_{\text {in }}(\Omega)\right)$.

Proof. Taking $v_{h}=2 P_{n-2} u_{h}$ in (1.5) and integrating $\left(\left(u_{h}\right)_{x x}, P_{n-2} u_{h}\right)=$ $\left(\left(u_{h}\right)_{x x}, u_{h}\right)$ by parts over $T$, we obtain

$$
\oint u_{h}^{2} \alpha \cdot n d \tau+2 \sigma\left\|\left(u_{h}\right)_{x}\right\|^{2}-2 \sigma \int_{\Gamma^{*}(T)}\left(u_{h}\right)_{x}^{-} u_{h} e_{1} \cdot n d \tau=2\left(f, u_{h}\right)+\gamma
$$

where

$\gamma=2\left(\left(u_{h}\right)_{\alpha}-f,\left(I-P_{n-2}\right) u_{h}\right)+2 \sigma \int_{\Gamma_{\text {in }}^{*}(T)}\left[\left(u_{h}\right)_{x}^{+}-\left(u_{h}\right)_{x}^{-}\right]\left(I-P_{n-2}\right) u_{h} e_{1} \cdot n d \tau$.

The integral over $\Gamma^{*}(T)$ reflects the fact that $e_{1} \cdot n=0$ on $\Gamma_{\text {in }}(\Omega)$. (A nonhorizontal $\Gamma_{i n}(\Omega)$ satisfying $(2.2)$ could be handled with a slightly modified form of (3.3).) Using (3.1) and the fact that $q$ is bounded, we obtain

$$
|\gamma| \leq C\left(\left\|\left(u_{h}\right)_{\alpha}\right\|_{T \cup I(T)}+\|f\|\right) \cdot\left(k\left\|\left(u_{h}\right)_{\alpha}\right\|+h\left\|\left(u_{h}\right)_{x}\right\|\right),
$$


where (2.6) has been applied to $\left(I-P_{n-2}\right) u_{h}$. Via the Schwarz and arithmeticgeometric mean inequalities:

$$
\begin{aligned}
|\gamma| \leq & C k\left(\left\|\left(u_{h}\right)_{\alpha}\right\|_{T \cup I(T)}^{2}+\|f\|^{2}\right) \\
& +\varepsilon \sigma\left\|\left(u_{h}\right)_{x}\right\|^{2}+C \frac{h^{2}}{\varepsilon \sigma}\left(\left\|\left(u_{h}\right)_{\alpha}\right\|_{T \cup I(T)}^{2}+\|f\|^{2}\right),
\end{aligned}
$$

where $\varepsilon>0$. Replacing $\frac{h^{2}}{\sigma}$ by $\frac{k}{q}$, we may write

$$
|\gamma| \leq \varepsilon \sigma\left\|\left(u_{h}\right)_{x}\right\|^{2}+\frac{C k}{\varepsilon q}\left((1+\varepsilon q)\left\|\left(u_{h}\right)_{\alpha}\right\|_{T \cup I(T)}^{2}+\|f\|^{2}\right) .
$$

Fixing $\varepsilon$ at a value less than 2 and substituting in (3.4), we obtain (3.3) with $\xi=2-\varepsilon$.

The stability result (3.3) will not stand alone because of the presence of $\left\|\left(u_{h}\right)_{r}\right\|_{T \cup I(T)}^{2}$ on the right-hand side. However, this is a quantity which we can control via the second test function

$$
v_{h}^{*} \equiv \frac{-2\left(u_{h}\right)_{\tau_{1} \tau_{2}}}{\left(\alpha \cdot n_{1}\right)\left(\alpha \cdot n_{2}\right)} .
$$

This test function approaches a scalar multiple of $\left(u_{h}\right)_{x x}$ as $q \rightarrow 0$, in a sense made precise by the following lemma.

Lemma 3.3. The test function $v_{h}^{*}$ satisfies

$$
v_{h}^{*}=-\frac{2}{\alpha_{2}^{2}}\left(u_{h}\right)_{x x}+\psi
$$

where

$$
\|\psi\| \leq C \frac{q}{\sigma}\left\|\left(u_{h}\right)_{\alpha}\right\| .
$$

Proof. If $v$ is a twice differentiable function on $T$,

$$
\left(\begin{array}{l}
v_{\tau_{1}} \\
v_{\tau_{2}}
\end{array}\right)=-\frac{1}{\alpha_{2}}\left(\begin{array}{ll}
\alpha \cdot n_{1} & n_{1,1} \\
\alpha \cdot n_{2} & n_{2,1}
\end{array}\right)\left(\begin{array}{l}
v_{x} \\
v_{\alpha}
\end{array}\right)
$$

Here, $n_{i}$ is the unit outer normal to side $\Gamma_{i}$ of $T$ and $n_{i, 1}$ is its first (horizontal) component. We thus have

$$
v_{\tau_{1}}=-\frac{1}{\alpha_{2}}\left[\left(\alpha \cdot n_{1}\right) v_{x}+n_{1,1} v_{\alpha}\right] .
$$

Differentiating with respect to $\tau_{2}$, we obtain

$$
v_{\tau_{1} \tau_{2}}=\frac{\left(\alpha \cdot n_{1}\right)\left(\alpha \cdot n_{2}\right)}{\alpha_{2}^{2}}\left[v_{x x}+\left(\frac{n_{2,1}}{\alpha \cdot n_{2}}+\frac{n_{1,1}}{\alpha \cdot n_{1}}\right) v_{\alpha x x}+\frac{n_{1,1}}{\alpha \cdot n_{1}} \frac{n_{2,1}}{\alpha \cdot n_{2}} v_{\alpha \alpha x}\right] .
$$

Hence, $v_{h}^{*}$ has the representation (3.5), where

$$
\psi=-\frac{2}{\alpha_{2}^{2}}\left[\left(\frac{n_{2,1}}{\alpha \cdot n_{2}}+\frac{n_{1,1}}{\alpha \cdot n_{1}}\right)\left(u_{h}\right)_{\alpha x}+\frac{n_{1,1}}{\alpha \cdot n_{1}} \frac{n_{2,1}}{\alpha \cdot n_{2}}\left(u_{h}\right)_{\alpha \alpha x}\right] .
$$


Applying assumption $\mathrm{H} 4$ and (2.2), then (2.4) and (2.5):

$$
\begin{aligned}
\|\psi\| & \leq C\left(\frac{k}{h}\left\|\left(u_{h}\right)_{\alpha . x}\right\|+\left(\frac{k}{h}\right)^{2}\left\|\left(u_{h}\right)_{\alpha \alpha x}\right\|\right) \\
& \leq C \frac{k}{h^{2}}\left\|\left(u_{h}\right)_{\alpha}\right\| \leq C \frac{q}{\sigma}\left\|\left(u_{h}\right)_{\alpha}\right\| . \quad \square
\end{aligned}
$$

Lemma 3.4. As in Figure 3.1, let $\Gamma_{3}(T)$ denote the inflow (outflow) side of a type I (type II) triangle, $\theta_{1}$ and $\theta_{2}$ the two interior angles of $T$ adjoining $\Gamma_{3}(T)$, and $n_{i}, i=1,2,3$, the unit outer normals to the three sides of $T$. For $v_{h}=v_{h}^{*}$, the inner products in (1.5) then satisfy the following relations, with $\varepsilon$ an arbitrary positive constant:

$$
\left(\left(u_{h}\right)_{\alpha}, v_{h}^{*}\right)=\oint \frac{\left(u_{h}\right)_{\tau}^{2}}{\alpha \cdot n} d \tau+\frac{\sin \theta_{1} \sin \theta_{2}}{\prod_{i=1}^{3}\left(\alpha \cdot n_{i}\right)}\left|\left(u_{h}\right)_{\alpha}\right|_{\Gamma_{3}(T)}^{2} .
$$

(ii) $\quad \sigma\left(\left(u_{h}\right)_{x x}, v_{h}^{*}\right)=-\frac{2 \sigma}{\alpha_{2}^{2}}\left\|\left(u_{h}\right)_{x x}\right\|^{2}+r$,

$$
\text { where }|r| \leq \varepsilon \sigma\left\|\left(u_{h}\right)_{x x}\right\|^{2}+C \frac{q^{2}}{\varepsilon \sigma}\left\|\left(u_{h}\right)_{\alpha}\right\|^{2} .
$$

(iii)

$$
\begin{aligned}
& \left|\sigma \int_{\Gamma_{\text {in }}^{*}(T)}\left[\left(u_{h}\right)_{x}^{+}-\left(u_{h}\right)_{x}^{-}\right] v_{h}^{*} e_{1} \cdot n d \tau\right| \\
& \leq \varepsilon \sigma\left\|\left(u_{h}\right)_{x x}\right\|^{2}+C \frac{q^{2}}{\sigma}\left(1+\frac{1}{\varepsilon}\right)\left\|\left(u_{h}\right)_{\alpha}\right\|_{T \cup I(T)}^{2} .
\end{aligned}
$$

(iv) $\quad\left|\left(f, v_{h}^{*}\right)\right| \leq \varepsilon \sigma\left\|\left(u_{h}\right)_{x x}\right\|^{2}$

$$
+C \sigma^{-1}\left(q^{2}\left\|\left(u_{h}\right)_{c x}\right\|^{2}+\left(1+\frac{1}{\varepsilon}\right)\left\|P_{n-2} f\right\|^{2}\right) .
$$

Proof. (i) This identity is proved in [1, p. 264]. We have made the substitutions $\tau_{1} \cdot n_{3}=-\sin \theta_{2}$ and $\tau_{2} \cdot n_{3}=\sin \theta_{1}$ in transcribing the result into the form (3.7).

(ii) From (3.5) and (3.6) we obtain (3.8) with

$$
|r|=\sigma\left|\left(\left(u_{h}\right)_{x x}, \psi\right)\right| \leq C q\left\|\left(u_{h}\right)_{x x}\right\| \cdot\left\|\left(u_{h}\right)_{\alpha}\right\|,
$$

from which the desired result follows, upon application of the Schwarz inequality.

(iii) Using (3.1) and Lemma 3.3,

$$
\begin{aligned}
& \left|\sigma \int_{\Gamma_{\text {in }}^{*}(T)}\left[\left(u_{h}\right)_{x}^{+}-\left(u_{h}\right)_{x}^{-}\right] v_{h}^{*} e_{1} \cdot n d \tau\right| \\
& \quad \leq C q\left\|\left(u_{h}\right)_{\alpha x}\right\|_{T \cup I(T)}\left(\frac{2}{\alpha_{2}^{2}}\left\|\left(u_{h}\right)_{x x}\right\|+\frac{q}{\sigma}\left\|\left(u_{h}\right)_{r}\right\|\right) .
\end{aligned}
$$

The bound (3.9) then follows from a suitable application of the arithmeticgeometric inequality to the product $\left\|\left(u_{h}\right)_{\alpha}\right\|_{T \cup I(T)} \cdot\left\|\left(u_{h}\right)_{x x}\right\|$. 
(iv) We have from Lemma 3.3:

$$
\left(f, v_{h}^{*}\right)=-\frac{2}{\alpha_{2}^{2}}\left(P_{n-2} f,\left(u_{h}\right)_{x x}\right)+\left(P_{n-2} f, \psi\right) \text {. }
$$

Thus,

$$
\begin{aligned}
\left|\left(f, v_{h}^{*}\right)\right| & \leq C\left\|\left(u_{h}\right)_{x x}\right\| \cdot\left\|P_{n-2} f\right\|+\|\psi\| \cdot\left\|P_{n-2} f\right\| \\
& \leq \varepsilon \sigma\left\|\left(u_{h}\right)_{x x}\right\|^{2}+\frac{C}{\varepsilon \sigma}\left\|P_{n-2} f\right\|^{2}+\sigma\|\psi\|^{2}+\frac{C}{\sigma}\left\|P_{n-2} f\right\|^{2} .
\end{aligned}
$$

The result follows from (3.6). it is:

We can now state the result of taking $v_{h}=v_{h}^{*}$ in (1.5). With $\varepsilon=\frac{1}{3}\left(\frac{2}{\alpha_{2}^{2}}-1\right)$,

$$
\begin{gathered}
\oint \frac{\left(u_{h}\right)_{\tau}^{2}}{\alpha \cdot n} d \tau+\frac{\sin \theta_{1} \sin \theta_{2}}{\prod_{i=1}^{3}\left(\alpha \cdot n_{i}\right)}\left|\left(u_{h}\right)_{\alpha}\right|_{\Gamma_{3}(T)}^{2}+\sigma\left\|\left(u_{h}\right)_{x x}\right\|^{2} \\
\leq C \sigma^{-1}\left(q^{2}\left\|\left(u_{h}\right)_{\alpha}\right\|_{T \cup I(T)}^{2}+\left\|P_{n-2} f\right\|^{2}\right) .
\end{gathered}
$$

With some additional work, we can recast this as follows:

Lemma 3.5. For sufficiently small $q, u_{h}$ satisfies

$$
\begin{aligned}
& \oint \frac{\left(u_{h}\right)_{\tau}^{2}}{\alpha \cdot n} d \tau+\mu \frac{q}{\sigma}\left\|\left(u_{h}\right)_{\alpha}\right\|^{2} \\
& \quad \leq C \sigma^{-1}\left(q^{2}\left\|\left(u_{h}\right)_{\alpha}\right\|_{I(T)}^{2}+\left\|P_{n-2} f\right\|^{2}+q\|f\|^{2}\right)
\end{aligned}
$$

where $\mu$ is a positive constant.

Proof. We first consider the case of a type I triangle $T$, for which $\left(u_{h}\right)_{\alpha}$ can be used as a test function. Upon application of (3.2), this choice leads to

$$
\left\|\left(u_{h}\right)_{,}\right\| \leq \sigma\left\|\left(u_{h}\right)_{x x}\right\|+C\left(q\left\|\left(u_{h}\right)_{\alpha}\right\|_{T \cup I(T)}+\|f\|\right) .
$$

By the arithmetic-geometric mean inequality,

$$
\left\|\left(u_{h}\right)_{\alpha r}\right\|^{2} \leq 2 \sigma^{2}\left\|\left(u_{h}\right)_{x x}\right\|^{2}+C\left(q^{2}\left\|\left(u_{h}\right)_{\alpha}\right\|_{T \cup I(T)}^{2}+\|f\|^{2}\right) .
$$

Hence,

$$
\sigma\left\|\left(u_{h}\right)_{x x}\right\|^{2} \geq \frac{1}{2 \sigma}\left\|\left(u_{h}\right)_{\alpha}\right\|^{2}-\frac{C}{\sigma}\left(q^{2}\left\|\left(u_{h}\right)_{\alpha}\right\|_{T \cup I(T)}^{2}+\|f\|^{2}\right) .
$$

Also, from assumptions $\mathrm{H} 3, \mathrm{H} 4$ and (2.3), we have

$$
\left|\frac{\sin \theta_{1} \sin \theta_{2}}{\prod_{i=1}^{3}\left(\alpha \cdot n_{i}\right)}\right|\left|\left(u_{h}\right)_{\alpha}\right|_{\Gamma_{3}(T)}^{2} \leq C^{\prime} \frac{q}{\sigma}\left\|\left(u_{h}\right)_{\alpha}\right\|^{2} .
$$

Now note that (3.11) remains valid upon replacement of $\sigma\left\|\left(u_{h}\right)_{x x}\right\|^{2}$ by $4 q C^{\prime} \sigma\left\|\left(u_{h}\right)_{x x}\right\|^{2}$ provided $q \leq \frac{1}{4 C^{\prime}}$. Substitution of (3.14) and (3.15) into (3.11) then yields $(3.12)$. 
We next assume that $T$ is of type II and take $v_{h}=P_{n-2}\left(u_{h}\right)_{\alpha}$ in (1.5). This yields, in analogy with (3.13) and (3.14):

$$
\left\|P_{n-2}\left(u_{h}\right)_{\alpha x}\right\|^{2} \leq 2 \sigma^{2}\left\|\left(u_{h}\right)_{x x}\right\|^{2}+C\left(q^{2}\left\|\left(u_{h}\right)_{\alpha}\right\|_{T \cup I(T)}^{2}+\left\|P_{n-2} f\right\|^{2}\right)
$$

and

$$
\sigma\left\|\left(u_{h}\right)_{x x}\right\|^{2} \geq \frac{1}{2 \sigma}\left\|P_{n-2}\left(u_{h}\right)_{\alpha}\right\|^{2}-\frac{C}{\sigma}\left(q^{2}\left\|\left(u_{h}\right)_{\alpha}\right\|_{T \cup I(T)}^{2}+\left\|P_{n-2} f\right\|^{2}\right) .
$$

Note that $\prod_{i=1}^{3}\left(\alpha \cdot n_{i}\right)$ is positive for a type II triangle; thus by (2.1),

$$
\frac{\sin \theta_{1} \sin \theta_{2}}{\prod_{i=1}^{3}\left(\alpha \cdot n_{i}\right)}\left|\left(u_{h}\right)_{\alpha}\right|_{\Gamma_{3}(T)}^{2} \geq \lambda \frac{q}{\sigma} k\left|\left(u_{h}\right)_{\alpha}\right|_{\Gamma_{3}(T)}^{2},
$$

where $\lambda$ is a positive constant. Combining (3.17) and (3.18), we obtain

$$
\begin{aligned}
& \frac{\sin \theta_{1} \sin \theta_{2}}{\prod_{i=1}^{3}\left(\alpha \cdot n_{i}\right)}\left|\left(u_{h}\right)_{\alpha}\right|_{\Gamma_{3}(T)}^{2}+\sigma\left\|\left(u_{h}\right)_{x x}\right\|^{2} \\
& \geq \frac{q}{\sigma}\left[\lambda k\left|\left(u_{h}\right)_{\alpha}\right|_{\Gamma_{3}(T)}^{2}+\frac{1}{2}\left\|P_{n-2}\left(u_{h}\right)_{\alpha}\right\|^{2}\right. \\
& \left.\quad-C\left(q^{2}\left\|\left(u_{h}\right)_{\alpha}\right\|_{T \cup I(T)}^{2}+\left\|P_{n-2} f\right\|^{2}\right)\right]
\end{aligned}
$$

provided $q \leq 1$. The following inequality is easily proved for $v_{h} \in \mathbf{P}_{n-1}(T)$ :

$$
\sqrt{k}\left|v_{h}\right|_{\Gamma_{3}(T)}+\left\|P_{n-2} v_{h}\right\| \geq \nu\left\|v_{h}\right\|,
$$

where $\nu$ is a positive constant. Thus $\lambda k\left|\left(u_{h}\right)_{\alpha}\right|_{\Gamma_{3}(T)}^{2}+\frac{1}{2}\left\|P_{n-2}\left(u_{h}\right)_{\alpha}\right\|^{2}$ in (3.19) coerces $\left\|\left(u_{h}\right)_{\alpha}\right\|^{2}$, and (3.11) again leads to the desired result for a type II triangle.

We are now ready to combine the results of Lemmas 3.2 and 3.5 into a stability result for a single triangle $T$ of either type. Multiplying (3.12) by $\frac{M h^{2}}{q}$, where $M$ is a constant to be specified below, and adding to (3.3), we obtain

$$
\begin{aligned}
& \oint\left[u_{h}^{2} \alpha \cdot n+\frac{M h^{2}}{q} \frac{\left(u_{h}\right)_{\tau}^{2}}{\alpha \cdot n}\right] d \tau+\xi \sigma\left\|\left(u_{h}\right)_{x}\right\|^{2} \\
& \quad-2 \sigma \oint\left(u_{h}\right)_{x}^{-} u_{h} e_{1} \cdot n d \tau+(M \mu-C) \frac{k}{q}\left\|\left(u_{h}\right)_{\alpha}\right\|^{2} \\
& \leq 2\left(f, u_{h}\right)+C \frac{k}{q}(M q+1)\left\|\left(u_{h}\right)_{(\alpha}\right\|_{I(T)}^{2} \\
& \quad+C \frac{k}{q}(1+M)\|f\|^{2}+C M \frac{k}{q^{2}}\left\|P_{n-2} f\right\|^{2},
\end{aligned}
$$

where the fact that $\frac{h^{2}}{\sigma}=\frac{k}{q}$ has been used. Without loss of generality, we may assume that $C$ has the same value wherever it appears in (3.21). In (3.21), the ratio of the coefficient of $\left\|\left(u_{h}\right)_{\alpha}\right\|_{I(T)}^{2}$ to that of $\left\|\left(u_{h}\right)_{\alpha}\right\|^{2}$ is

$$
\frac{C(M q+1)}{M \mu-C}
$$


which can be made smaller than any given $\varepsilon>0$ by taking $q \leq \frac{1}{M}$, where $M=\frac{C}{\mu}\left(1+\frac{2}{\varepsilon}\right)$. With these stipulations we may write

$$
\begin{aligned}
\oint\left[u_{h}^{2} \alpha \cdot n+\frac{M h^{2}}{q} \frac{\left(u_{h}\right)_{\tau}^{2}}{\alpha \cdot n}\right] d \tau+\xi \sigma\left\|\left(u_{h}\right)_{x}\right\|^{2}+N \frac{k}{q}\left\|\left(u_{h}\right)_{\alpha}\right\|^{2} \\
\quad-2 \sigma \int_{\Gamma^{*}(T)}\left(u_{h}\right)_{x}^{-} u_{h} e_{1} \cdot n d \tau \\
\quad \leq 2\left(f, u_{h}\right)+\epsilon N \frac{k}{q}\left\|\left(u_{h}\right)_{\alpha}\right\|_{I(T)}^{2}+C \frac{k}{q}\left(\|f\|^{2}+\frac{1}{q}\left\|P_{n-2} f\right\|^{2}\right),
\end{aligned}
$$

where $N$ is a positive constant.

We can now establish a global stability result:

Theorem 3.1. There exist positive constants $M, q_{0}, \xi, \eta$ such that for $q \leq q_{0}$,

$$
\begin{gathered}
\int_{\Gamma_{\text {oul }}(\Omega)}\left[u_{h}^{2}|\alpha \cdot n|+\frac{M h^{2}}{q} \frac{\left(u_{h}\right)_{\tau}^{2}}{|\alpha \cdot n|}\right] d \tau+\xi \sigma\left\|\left(u_{h}\right)_{x}\right\|_{\Omega}^{2}+\eta \frac{k}{q}\left\|\left(u_{h}\right)_{\alpha}\right\|_{\Omega}^{2} \\
\leq \int_{\Gamma_{\text {in }}(\Omega)}\left[u_{h}^{2}|\alpha \cdot n|+\frac{M h^{2}}{q} \frac{\left(u_{h}\right)_{\tau}^{2}}{|\alpha \cdot n|}\right] d \tau \\
+2\left(f, u_{h}\right)_{\Omega}+C \frac{k}{q}\left(\|f\|_{\Omega}^{2}+\frac{1}{q}\left\|P_{n-2} f\right\|_{\Omega}^{2}\right) .
\end{gathered}
$$

Proof. For any triangle $T, I(T)$ consists of at most two triangles, so that by taking $\varepsilon<\frac{1}{2}$ in (3.22), the term $\varepsilon N \frac{k}{q}\left\|\left(u_{h}\right)_{\alpha}\right\|_{I(T)}^{2}$ can be absorbed by the corresponding term on the left-hand side when (3.22) is summed over all triangles. Such summation also yields the term $-2 \sigma \int_{\Gamma_{\text {ou }}(\Omega)}\left(u_{h}\right)_{x}^{-} u_{h} e_{1} \cdot n d \tau$, which has been absorbed by the left-hand side of (3.23). For, by (2.2) and (2.3), we have

$$
\begin{aligned}
\left|2 \sigma \int_{\Gamma_{\text {out }}(\Omega)}\left(u_{h}\right)_{x}^{-} u_{h} e_{1} \cdot n d \tau\right| & \leq C \sigma \frac{\sqrt{k}}{h}\left\|\left(u_{h}\right)_{x}\right\|_{\Omega}\left|u_{h}\right|_{\Gamma_{\text {out }}(\Omega)} \\
& \leq \varepsilon^{\prime}\left|u_{h}\right|_{\Gamma_{\text {out }}(\Omega)}^{2}+\frac{C}{\varepsilon^{\prime}} q \sigma\left\|\left(u_{h}\right)_{x}\right\|_{\Omega}^{2},
\end{aligned}
$$

where $\varepsilon^{\prime}>0$. Taking first $\varepsilon^{\prime}$, then $q$, sufficiently small, we obtain (3.23) (for different $M, q_{0}, \xi$ than in (3.22)).

Note that in the above proof, we have not used the fact that $e_{1} \cdot n=0$ on $\Gamma_{\text {out }}(\Omega)$. The result therefore holds not just over an entire triangulation of $\Omega$ but also over any subset of triangles $\Omega^{\prime}$ for which $\Gamma_{\text {in }}\left(\Omega^{\prime}\right) \subset \Gamma_{\text {in }}(\Omega)$ (in which case $u_{h}$ can be developed in $\Omega^{\prime}$ independently of $\Omega-\Omega^{\prime}$ ).

\section{ERROR ESTIMATES}

We now use the local stability result (3.23) to obtain global error estimates. The exact solution to (1.1) satisfies the same inner product relations (1.5) as 
$u_{h} ;$ thus for all $v_{h} \in \mathbf{P}_{n-\rho(T)}(T)$,

$$
\begin{aligned}
& \left(\left(u_{h}-u\right)_{\alpha}-\sigma\left(u_{h}-u\right)_{x x}, v_{h}\right) \\
& \quad+\sigma \int_{\Gamma_{\text {in }}^{*}(T)}\left[\left(u_{h}-u\right)_{x}^{+}-\left(u_{h}-u\right)_{x}^{-}\right] v_{h} e_{1} \cdot n d \tau=0 .
\end{aligned}
$$

Let $u_{I}$ denote a continuous piecewise $n$th degree interpolant for $u$ over the triangulation, and $e_{h} \equiv u_{h}-u_{I}$. From (4.1) we obtain

$$
\left(\left(e_{h}\right)_{\alpha x}-\sigma\left(e_{h}\right)_{x x}, v_{h}\right)+\sigma \int_{\Gamma_{\text {in }}^{*}(T)}\left[\left(e_{h}\right)_{x}^{+}-\left(e_{h}\right)_{x}^{-}\right] v_{h} e_{1} \cdot n d \tau=\left(r+s, v_{h}\right)
$$

where

$$
r=\left(u-u_{I}\right)_{\alpha}-\sigma\left(u-u_{I}\right)_{x x}
$$

and $s \in \mathbf{P}_{n-\rho(T)}(T)$ is defined by

$$
\left(s, v_{h}\right)=-\sigma \int_{\Gamma_{\mathrm{in}}^{*}(T)}\left[\left(u_{I}\right)_{x}^{+}-\left(u_{I}\right)_{x}^{-}\right] v_{h} e_{1} \cdot n d \tau, \quad \text { all } v_{h} \in \mathbf{P}_{n-\rho(T)}(T) .
$$

As in [1], we define $u_{I}$ as follows:

(i) $\quad u_{I}=u$ at the vertices of $T$.

(ii) On each side $\Gamma_{i}$ of $T$,

$$
\int_{\Gamma_{i}}\left(u-u_{I}\right) v d \tau=0 \text { for polynomials } v(\tau) \text { of degree } \leq n-2 \text {. }
$$

(iii) $\quad \iint_{T}\left(u-u_{I}\right) w d x d y=0$ for polynomials $w \in \mathbf{P}_{n-3}(T)$.

The following error estimates hold for $u_{l}$, where \|\|$_{n+1}$ denotes the norm on $H^{n+1}(T)$ :

$$
\begin{aligned}
\left\|u-u_{I}\right\| & \leq C h^{n+1}\|u\|_{n+1}, \\
\left\|\left(u-u_{I}\right)_{\alpha}\right\| & \leq C k^{-1} h^{n+1}\|u\|_{n+1}, \\
\left\|\left(u-u_{I}\right)_{x x}\right\| & \leq C h^{n-1}\|u\|_{n+1}, \\
\left|u-u_{I}\right|_{\Gamma(T)} & \leq C k^{-1 / 2} h^{n+1}\|u\|_{n+1}, \\
\left|\left(u-u_{I}\right)_{x}\right|_{\Gamma(T)} & \leq C k^{-1 / 2} h^{n}\|u\|_{n+1} .
\end{aligned}
$$

Note also that

$$
P_{n-2}\left(u-u_{l}\right)_{\alpha}=0
$$


since integration by parts yields

$$
\left(\left(u-u_{I}\right)_{\alpha}, v_{h}\right)=\oint\left(u-u_{I}\right) v_{h} \alpha \cdot n d \tau-\left(u-u_{I},\left(v_{h}\right)_{\alpha}\right)
$$

and the terms on the right vanish for $v_{h} \in \mathbf{P}_{n-2}(T)$ by conditions (ii) and (iii) above.

Applying (3.23) to (4.2), we obtain

$$
\begin{aligned}
\int_{\Gamma_{\text {oul }}(\Omega)}\left[e_{h}^{2}|\alpha \cdot n|+\frac{M h^{2}}{q} \frac{\left(e_{h}\right)_{\tau}^{2}}{|\alpha \cdot n|}\right] d \tau+\xi \sigma\left\|\left(e_{h}\right)_{x}\right\|_{\Omega}^{2}+\eta \frac{k}{q}\left\|\left(e_{h}\right)_{\alpha}\right\|_{\Omega}^{2} \\
\leq \int_{\Gamma_{\text {in }}(\Omega)}\left[e_{h}^{2}|\alpha \cdot n|+\frac{M h^{2}}{q} \frac{\left(e_{h}\right)_{\tau}^{2}}{|\alpha \cdot n|}\right] d \tau \\
+2\left(r+s, e_{h}\right)_{\Omega}+C \frac{k}{q}\left(\|r+s\|_{\Omega}^{2}+\frac{1}{q}\left\|P_{n-2}(r+s)\right\|_{\Omega}^{2}\right) .
\end{aligned}
$$

Lemma 4.1. For arbitrary $\varepsilon>0$,

$$
\begin{aligned}
2\left(r+s, e_{h}\right)_{\Omega}+C \frac{k}{q}\left(\|r+s\|_{\Omega}^{2}+\frac{1}{q}\left\|P_{n-2}(r+s)\right\|_{\Omega}^{2}\right) \\
\leq \varepsilon\left(\left|e_{h}\right|_{\Gamma_{\text {out }}(\Omega)}^{2}+\sigma\left\|\left(e_{h}\right)_{x}\right\|_{\Omega}^{2}+\frac{k}{q}\left\|\left(e_{h}\right)_{\alpha}\right\|_{\Omega}^{2}\right) \\
+C\left(1+\frac{q}{\varepsilon}\right) \frac{\sigma}{q^{2}} h^{2 n}\|u\|_{n+1, \Omega}^{2} .
\end{aligned}
$$

Proof. From (4.4)-(4.8),

$$
\begin{aligned}
\left\|P_{n-2} r\right\|_{\Omega} & \leq C q k^{-1} h^{n+1}\|u\|_{n+1, \Omega} \\
\|r\|_{\Omega} & \leq C k^{-1} h^{n+1}\|u\|_{n+1, \Omega}, \\
\|s\|_{\Omega} & \leq C q k^{-1} h^{n+1}\|u\|_{n+1, \Omega}
\end{aligned}
$$

Thus,

$$
\|r+s\|_{\Omega}^{2}+\frac{1}{q}\left\|P_{n-2}(r+s)\right\|_{\Omega}^{2} \leq C k^{-2} h^{2 n+2}\|u\|_{n+1, \Omega}^{2} .
$$

Integration by parts yields

$$
\begin{aligned}
\left(r+s, e_{h}\right)_{\Omega}= & \oint_{\Gamma(\Omega)}\left(u-u_{I}\right) e_{h} \alpha \cdot n d \tau-\sigma \int_{\Gamma_{\text {out }}(\Omega)}\left(u-u_{I}\right)_{x}^{-} e_{h} e_{1} \cdot n d \tau \\
& +\sigma\left(\left(u-u_{I}\right)_{x},\left(e_{h}\right)_{x}\right)_{\Omega}-\left(u-u_{I},\left(e_{h}\right)_{\alpha}\right)_{\Omega},
\end{aligned}
$$


and the terms on the right can be bounded as follows for arbitrary $\varepsilon>0$ :

$$
\begin{aligned}
& \oint_{\Gamma(\Omega)}\left(u-u_{I}\right) e_{h} \alpha \cdot n d \tau \leq \frac{1}{2} \varepsilon\left|e_{h}\right|_{\Gamma(\Omega)}^{2}+\frac{C}{\varepsilon}\left|u-u_{I}\right|_{\Gamma(\Omega)}^{2} \\
& \leq \frac{1}{2} \varepsilon\left|e_{h}\right|_{\Gamma(\Omega)}^{2}+\frac{C}{\varepsilon} \frac{\sigma}{q} h^{2 n}\|u\|_{n+1, \Omega}^{2}, \\
& \sigma \int_{\Gamma_{\text {out }}(\Omega)}\left(u-u_{I}\right)_{x}^{-} e_{h} e_{1} \cdot n d \tau \leq \frac{1}{2} \varepsilon\left|e_{h}\right|_{\Gamma_{\text {out }}(\Omega)}^{2}+\frac{C}{\varepsilon}\left(\frac{\sigma k}{h}\right)^{2}\left|\left(u-u_{I}\right)_{x}\right|_{\Gamma_{\text {out }}(\Omega)}^{2} \\
& \leq \frac{1}{2} \varepsilon\left|e_{h}\right|_{\Gamma_{\text {oul }}(\Omega)}^{2}+\frac{C}{\varepsilon} q \sigma h^{2 n}\|u\|_{n+1, \Omega}^{2}, \\
& \sigma\left(\left(u-u_{I}\right)_{x},\left(e_{h}\right)_{x}\right)_{\Omega} \leq \sigma\left(\varepsilon\left\|\left(e_{h}\right)_{x}\right\|_{\Omega}^{2}+\frac{C}{\varepsilon}\left\|\left(u-u_{I}\right)_{x}\right\|_{\Omega}^{2}\right) \\
& \leq \varepsilon \sigma\left\|\left(e_{h}\right)_{x}\right\|_{\Omega}^{2}+\frac{C}{\varepsilon} \sigma h^{2 n}\|u\|_{n+1, \Omega}^{2} \\
& \left(u-u_{I},\left(e_{h}\right)_{\alpha}\right)_{\Omega} \leq \varepsilon \frac{k}{q}\left\|\left(e_{h}\right)_{\alpha}\right\|_{\Omega}^{2}+\frac{C}{\varepsilon} \frac{q}{k}\left\|u-u_{I}\right\|_{\Omega}^{2} \\
& \leq \varepsilon \frac{k}{q}\left\|\left(e_{h}\right)_{\alpha}\right\|_{\Omega}^{2}+\frac{C}{\varepsilon} \sigma h^{2 n}\|u\|_{n+1, \Omega}^{2} .
\end{aligned}
$$

When the above inequalities are added to $C \frac{k}{q}$ times (4.12), and $\varepsilon$ replaced by $\frac{\varepsilon}{2}$, the desired result follows.

We can now obtain global error estimates by taking $\varepsilon$ sufficiently small in (4.10) and combining with (4.9). Assuming an optimal-order interpolant has been chosen for $u_{h}$ on $\Gamma_{i n}(\Omega)$, we have (for different constants than in (4.9)):

Theorem 4.1. There exist positive constants $M, q_{0}, \xi, \eta$ such that for $q \leq q_{0}$ the error $e_{h}$ in the finite element scheme satisfies

$$
\begin{gathered}
\int_{\Gamma_{\text {out }}(\Omega)}\left[e_{h}^{2}|\alpha \cdot n|+\frac{M h^{2}}{q} \frac{\left(e_{h}\right)_{\tau}^{2}}{|\alpha \cdot n|}\right] d \tau+\xi \sigma\left\|\left(e_{h}\right)_{x}\right\|_{\Omega}^{2}+\eta \frac{k}{q}\left\|\left(e_{h}\right)_{\alpha}\right\|_{\Omega}^{2} \\
\leq C \frac{\sigma}{q^{2}} h^{2 n}\|u\|_{n+1, \Omega}^{2} .
\end{gathered}
$$

Writing $e_{h}=\left(u_{h}-u\right)-\left(u_{I}-u\right)$ and using the fact that $u_{I}$ achieves optimalorder accuracy, we conclude:

\section{Corollary 4.1. There holds}

$$
\begin{aligned}
& \left|u-u_{h}\right|_{\Gamma_{\text {out }}(\Omega)} \leq C \frac{\sqrt{\sigma} h^{n}}{q}\|u\|_{n+1, \Omega}, \\
& \left\|\left(u-u_{h}\right)_{x}\right\|_{\Omega} \leq C \frac{h^{n}}{q}\|u\|_{n+1, \Omega}, \\
& \left\|\left(u-u_{h}\right)_{\alpha}\right\|_{\Omega} \leq C \frac{\sigma h^{n-1}}{q}\|u\|_{n+1, \Omega} .
\end{aligned}
$$

Some additional work is needed to derive the estimate (1.7). As in [1], we may think of $u_{h}$ as evolving in "layers" of triangles. For a given triangulation 
$\{T\}$, we define

$$
\begin{aligned}
& S_{0}=\varnothing, \\
& S_{i}=\left\{T \mid \Gamma_{\text {in }}(T) \subset \Gamma_{\text {in }}\left(\Omega-\bigcup_{j<i} S_{j}\right)\right\}, \quad i=1,2, \ldots .
\end{aligned}
$$

The approximate solution can be computed in parallel in the triangles of $S_{1}$, then $S_{2}$, etc. After $u_{h}$ has been computed in $S_{i}$, it has advanced to the "frontline"

$$
F_{i} \equiv \Gamma_{\text {in }}(\Omega)-\Gamma_{\text {in }}\left(\bigcup_{j \leq i} S_{j}\right) \cup \Gamma_{\text {out }}\left(\bigcup_{j \leq i} S_{j}\right) .
$$

Using (2.13) with $u_{h}$ replaced by $e_{h}$ and $f$ by $r+s$, then extending the result to a layer, we obtain

$$
\left\|e_{h}\right\|_{S_{i}}^{2} \leq C\left(k\left|e_{h}\right|_{F_{i-1}}^{2}+q^{2} h^{2} k\left|\left(e_{h}\right)_{x}^{-}\right|_{F_{i-1}}^{2}+k^{2}\|r+s\|_{S_{i}}^{2}\right),
$$

with the $\left(e_{h}\right)_{x}^{-}$term omitted for $i=1$. Via (2.3), $\left|\left(e_{h}\right)_{x}^{-}\right|_{F_{i-1}}^{2} \leq C k^{-1}\left\|\left(e_{h}\right)_{x}\right\|_{S_{i-1}}^{2}$ for $i \neq 1$. Summation of (4.17) over all layers thus gives

$$
\begin{aligned}
\left\|e_{h}\right\|_{\Omega}^{2} & \leq C\left(k \sum_{i}\left|e_{h}\right|_{F_{i-1}}^{2}+q^{2} h^{2}\left\|\left(e_{h}\right)_{x}\right\|_{\Omega}^{2}+k^{2}\|r+s\|_{\Omega}^{2}\right) \\
& \leq C\left(k \sum_{i}\left|e_{h}\right|_{F_{i-1}}^{2}+h^{2 n+2}\|u\|_{n+1, \Omega}^{2}\right),
\end{aligned}
$$

using (4.12) and (4.15).

Applying Theorem 4.1 to $\Omega_{i} \equiv \bigcup_{j \leq i} S_{j}$ rather than all of $\Omega$, we infer

$$
\int_{F_{i}} e_{h}^{2}|\alpha \cdot n| d \tau \leq C \frac{\sigma h^{2 n}}{q^{2}}\|u\|_{n+1, \Omega_{i}}^{2} .
$$

Thus:

Corollary 4.2. Assuming there are $O\left(\frac{1}{k}\right)$ layers per triangulation,

$$
\left\|u-u_{h}\right\|_{\Omega} \leq C \sqrt{\sigma} q^{-1} h^{n}\|u\|_{n+1, \Omega}
$$

We summarize some special cases of the error estimates (4.14)-(4.16) and (4.19). 1) For constant $\sigma>0$, independent of $h$, we obtain $O\left(h^{n}\right)$ convergence for $u_{h}$ and $\left(u_{h}\right)_{x}$ and $O\left(h^{n-1}\right)$ for $\left(u_{h}\right)_{\alpha}$. Both derivative estimates are of optimal order on a mesh for which $k=O\left(h^{2}\right)$. 2) For $\sigma$ proportional to $h$ as $h \rightarrow 0$, the stability condition allows $k$ to be chosen in proportion to $h$. Here we obtain $O\left(h^{n+1 / 2}\right)$ convergence for $u_{h}$ and $O\left(h^{n}\right)$ (optimal) for $\left(u_{h}\right)_{x}$ and $\left(u_{h}\right)_{1} .3$ ) If $\sigma<O(h)$ as $h \rightarrow 0$ (i.e., $\frac{\sigma}{h} \rightarrow 0$ ), we would still want to take $k$ proportional to $h$ for approximability, causing $q \rightarrow 0$ as $h \rightarrow 0$. The estimate for $\left(u_{h}\right)_{\text {r }}$ remains of optimal order; however, the others deteriorate. A threshold is $\sigma=O\left(h^{3 / 2}\right)$, for which the estimates for $u_{h}$ and $\left(u_{h}\right)_{x}$ have 
declined to $O\left(h^{n+1 / 4}\right)$ and $O\left(h^{n-1 / 2}\right)$, the same as those previously obtained in [1] for the hyperbolic limit. In the next section, we show that these same estimates hold in the gap $\sigma \in\left(0, O\left(h^{3 / 2}\right)\right)$.

\section{THE CONVECTION-DOMINATED CASE}

We now extend the preceding analysis to the hyperbolic limit of (1.1). In this section, we assume $0 \leq \sigma<O(h)$ as $h \rightarrow 0$ and consider a family of triangulations for which the aspect ratio $\frac{k}{h}$ is uniformly bounded away from zero and infinity. In this setting, assumption $\mathrm{H} 2$ is the standard minimum angle condition, $\mathrm{H} 3$ is vacuous, and $q \rightarrow 0$ as $h \rightarrow 0$.

In place of Lemma 3.2 we derive the following result, which remains well behaved regardless of the size of $\sigma$.

Lemma 5.1. For $h$ (thus $q$ ) sufficiently small and arbitrary $\varepsilon>0$,

$$
\begin{aligned}
& \oint u_{h}^{2} \alpha \cdot n d \tau+2 \sigma\left\|\left(u_{h}\right)_{x}\right\|^{2}-2 \sigma \int_{\Gamma^{*}(T)}\left(u_{h}\right)_{x}^{-} u_{h} e_{1} \cdot n d \tau \\
& \leq \varepsilon h^{1 / 2}\left\|\left(u_{h}\right)_{\alpha}\right\|_{T \cup I(T)}^{2} \\
&+C\left(\left\|P_{n-2} f\right\|^{2}+\frac{h^{3 / 2}}{\varepsilon}\|f\|^{2}+h\left|u_{h}\right|_{\Gamma_{\text {in }}(T)}^{2}+\frac{h^{5 / 2}}{\varepsilon}\left|\left(u_{h}\right)_{\tau}\right|_{\Gamma_{\text {in }}(T)}^{2}\right) .
\end{aligned}
$$

Proof. Analogously to the proof of Lemma 3.2, we have

$$
\oint u_{h}^{2} \alpha \cdot n d \tau+2 \sigma\left\|\left(u_{h}\right)_{x}\right\|^{2}-2 \sigma \int_{\Gamma^{*}(T)}\left(u_{h}\right)_{x}^{-} u_{h} e_{1} \cdot n d \tau=\gamma,
$$

where

$$
\begin{aligned}
|\gamma| & \leq 2\left(P_{n-2} f, u_{h}\right)+C\left\|\left(u_{h}\right)_{\alpha}\right\|_{T \cup I(T)} \cdot\left\|\left(I-P_{n-2}\right) u_{h}\right\| \\
& \leq 2\left\|P_{n-2} f\right\| \cdot\left\|u_{h}\right\|+C h\left\|\left(u_{h}\right)_{\alpha}\right\|_{T \cup I(T)} \cdot\left\|\nabla u_{h}\right\| \\
& \leq \varepsilon h^{1 / 2}\left\|\left(u_{h}\right)_{\alpha}\right\|_{T \cup I(T)}^{2}+\left\|u_{h}\right\|^{2}+C\left(\left\|P_{n-2} f\right\|^{2}+\frac{h^{3 / 2}}{\varepsilon}\left\|\nabla u_{h}\right\|^{2}\right) .
\end{aligned}
$$

The desired result then follows by applying the bounds

$$
\left\|u_{h}\right\| \leq C\left(\sqrt{h}\left|u_{h}\right|_{\Gamma_{\text {in }}(T)}+q h\left\|\left(u_{h}\right)_{\alpha}\right\|_{I(T)}+q h^{3 / 2}\left|\left(u_{h}\right)_{\tau}\right|_{\Gamma_{\text {in }}^{*}(T)}+h\|f\|\right)
$$

and

$$
\left\|\nabla u_{h}\right\| \leq C\left(\sqrt{h}\left|\left(u_{h}\right)_{\tau}\right|_{\Gamma_{\text {in }}(T)}+q\left\|\left(u_{h}\right)_{\alpha}\right\|_{I(T)}+\|f\|\right) .
$$

The first of these can be obtained by taking $k=h$ in (2.13), then using the bound

$$
\left|\left(u_{h}\right)_{x}^{-}\right|_{\Gamma_{\text {in }}^{*}(T)} \leq C\left(\left|\left(u_{h}\right)_{\tau}\right|_{\Gamma_{\text {in }}^{*}(T)}+\frac{1}{\sqrt{h}}\left\|\left(u_{h}\right)_{\alpha}\right\|_{I(T)}\right),
$$

which arises from assumption $\mathrm{H} 4$ and (2.3). The second can be shown by writing $u_{h}=u_{h}(P)+w_{h}$ for fixed $P \in \Gamma_{\text {in }}(T)$ and observing that $w_{h}$ satisfies the same inner product relations (1.5) as $u_{h}$. Applying (5.3) to $w_{h}$, then using the inequalities

$$
\left\|\nabla u_{h}\right\|=\left\|\nabla w_{h}\right\| \leq C h^{-1}\left\|w_{h}\right\|
$$


and

gives (5.4).

$$
\left|w_{h}\right|_{\Gamma_{\text {in }}(T)} \leq C h\left|\left(w_{h}\right)_{\tau}\right|_{\Gamma_{\text {in }}(T)}=C h\left|\left(u_{h}\right)_{\tau}\right|_{\Gamma_{\text {in }}(T)}
$$

We next establish a convection-dominated counterpart of Lemma 3.5.

Lemma 5.2. There exists a constant $\mu>0$ such that for $h$ sufficiently small,

$$
\begin{aligned}
& \oint \frac{\left(u_{h}\right)_{\tau}^{2}}{\alpha \cdot n} d \tau+\frac{\mu}{h}\left\|\left(u_{h}\right)_{\alpha}\right\|^{2} \\
& \quad \leq C\left(\frac{\sigma}{h^{2}}\left\|\left(u_{h}\right)_{\alpha}\right\|_{I(T)}^{2}+h\left|\left(u_{h}\right)_{\tau}\right|_{\Gamma_{\text {in }}(T)}^{2}+h^{-2}\left\|P_{n-2} f\right\|^{2}+h^{-1}\|f\|^{2}\right) .
\end{aligned}
$$

Proof. In place of (3.10), we derive a bound which does not degrade as $\sigma \rightarrow 0$ :

$$
\begin{aligned}
\left|\left(f, v_{h}^{*}\right)\right| & \leq C\left\|\left(u_{h}\right)_{\tau_{1} \tau_{2}}\right\| \cdot\left\|P_{n-2} f\right\| \\
& \leq C h^{-1}\left\|\nabla u_{h}\right\| \cdot\left\|P_{n-2} f\right\| \\
& \leq C\left(\left\|\nabla u_{h}\right\|^{2}+h^{-2}\left\|P_{n-2} f\right\|^{2}\right) \\
& \leq C\left(h\left|\left(u_{h}\right)_{\tau}\right|_{\Gamma_{\text {in }}(T)}^{2}+q^{2}\left\|\left(u_{h}\right)_{\alpha}\right\|_{I(T)}^{2}+h^{-2}\left\|P_{n-2} f\right\|^{2}+\|f\|^{2}\right),
\end{aligned}
$$

where (5.4) was used in the last of the above inequalities. Equations (3.7)-(3.9) and (5.6) now yield the following analog of (3.11):

$$
\begin{aligned}
& \oint \frac{\left(u_{h}\right)_{\tau}^{2}}{\alpha \cdot n} d \tau+\frac{\sin \theta_{1} \sin \theta_{2}}{\prod_{i=1}^{3}\left(\alpha \cdot n_{i}\right)}\left|\left(u_{h}\right)_{\alpha}\right|_{\Gamma_{3}(T)}^{2}+\sigma\left\|\left(u_{h}\right)_{x x}\right\|^{2} \\
& \quad \leq C\left(\frac{\sigma}{h^{2}}\left\|\left(u_{h}\right)_{\alpha}\right\|_{T \cup I(T)}^{2}+h\left|\left(u_{h}\right)_{\tau}\right|_{\Gamma_{\text {in }}(T)}^{2}+h^{-2}\left\|P_{n-2} f\right\|^{2}+\|f\|^{2}\right) .
\end{aligned}
$$

To complete the proof for a type I triangle, we add $\frac{1}{\varepsilon h}$ times (3.13) to (5.7), apply (3.15), then fix $\varepsilon$ at a suitably small positive value. For a type II triangle, we add $\frac{1}{h}$ times (3.16) to (5.7), then use (3.18) and (3.20).

Adding $h^{3 / 2}$ times (5.5) to (5.1), we obtain

$$
\begin{aligned}
& \int_{\Gamma_{\text {out }}(T)}\left[u_{h}^{2}|\alpha \cdot n|+h^{3 / 2} \frac{\left(u_{h}\right)_{\tau}^{2}}{|\alpha \cdot n|}\right] d \tau+(\mu-\varepsilon) \sqrt{h}\left\|\left(u_{h}\right)_{\alpha}\right\|^{2}+2 \sigma\left\|\left(u_{h}\right)_{x}\right\|^{2} \\
& \quad-2 \sigma \int_{\Gamma^{*}(T)}\left(u_{h}\right)_{x}^{-} u_{h} e_{1} \cdot n d \tau \\
& \leq(1+(1+1 / \varepsilon) O(h)) \int_{\Gamma_{\text {in }}(T)}\left[u_{h}^{2}|\alpha \cdot n|+h^{3 / 2} \frac{\left(u_{h}\right)_{\tau}^{2}}{|\alpha \cdot n|}\right] d \tau \\
& +C\left(\left(\frac{\sigma}{h}+\varepsilon\right) \sqrt{h}\left\|\left(u_{h}\right)_{\alpha}\right\|_{I(T)}^{2}+h^{-1 / 2}\left\|P_{n-2} f\right\|^{2}+h^{1 / 2}(1+1 / \varepsilon)\|f\|^{2}\right) .
\end{aligned}
$$

This result generalizes directly to an entire layer of triangles. If we now take $\varepsilon$ suitably small and sum layer by layer, the $\left\|\left(u_{h}\right)_{\alpha}\right\|_{I(T)}$ terms on the right can be 
cancelled by the $\left\|\left(u_{h}\right)_{\alpha}\right\|$ terms on the left provided $h$ is sufficiently small. We thus obtain

$$
\begin{aligned}
\int_{\Gamma_{\text {out }}(\Omega)} & {\left[u_{h}^{2}|\alpha \cdot n|+h^{3 / 2} \frac{\left(u_{h}\right)_{\tau}^{2}}{|\alpha \cdot n|}\right] d \tau+\mu^{\prime} \sqrt{h}\left\|\left(u_{h}\right)_{\alpha}\right\|_{\Omega}^{2}+2 \sigma\left\|\left(u_{h}\right)_{x}\right\|_{\Omega}^{2} } \\
& -2 \sigma \int_{\Gamma_{\text {out }}(\Omega)}\left(u_{h}\right)_{x}^{-} u_{h} e_{1} \cdot n d \tau \\
\leq & C\left(\int_{\Gamma_{\text {in }}(\Omega)}\left[u_{h}^{2}|\alpha \cdot n|+h^{3 / 2} \frac{\left(u_{h}\right)_{\tau}^{2}}{|\alpha \cdot n|}\right] d \tau\right. \\
& \left.+h^{-1 / 2}\left\|P_{n-2} f\right\|_{\Omega}^{2}+h^{1 / 2}\|f\|_{\Omega}^{2}\right),
\end{aligned}
$$

where $\mu^{\prime}$ is a positive constant. Moreover,

$$
\begin{aligned}
& \left|\sigma \int_{\Gamma_{\text {out }}(\Omega)}\left(u_{h}\right)_{x}^{-} u_{h} e_{1} \cdot n d \tau\right| \leq C \sigma\left(\left|\nabla u_{h}\right|_{\Gamma_{\text {out }}(\Omega)}\left|u_{h}\right|_{\Gamma_{\text {out }}(\Omega)}\right) \\
& \quad \leq C \sigma\left(\left|\left(u_{h}\right)_{\tau}\right|_{\Gamma_{\text {out }}(\Omega)}+h^{-1 / 2}\left\|\left(u_{h}\right)_{\alpha}\right\|_{\Omega}\right)\left|u_{h}\right|_{\Gamma_{\text {out }}(\Omega)} \\
& \quad \leq \varepsilon\left|u_{h}\right|_{\Gamma_{\text {out }}(\Omega)}^{2}+C \frac{\sigma^{2}}{\varepsilon}\left(\left|\left(u_{h}\right)_{\tau}\right|_{\Gamma_{\text {out }}(\Omega)}^{2}+h^{-1}\left\|\left(u_{h}\right)_{\alpha \gamma}\right\|_{\Gamma_{\text {out }}(\Omega)}^{2}\right) \\
& \quad \leq \varepsilon\left|u_{h}\right|_{\Gamma_{\text {out }}(\Omega)}^{2}+\frac{C}{\varepsilon}\left(h^{2}\left|\left(u_{h}\right)_{\tau}\right|_{\Gamma_{\text {out }}(\Omega)}^{2}+h\left\|\left(u_{h}\right)_{\alpha}\right\|_{\Omega}^{2}\right),
\end{aligned}
$$

since $\sigma<O(h)$. Fixing $\varepsilon$ at a suitably small value in (5.10), we see that for $h$ sufficiently small, the term $-2 \sigma \int_{\Gamma_{\text {out }}(\Omega)}\left(u_{h}\right)_{x}^{-} u_{h} e_{1} \cdot n d \tau$ in (5.9) can be absorbed by other terms on the left-hand side of (5.9). This leads to the stability result:

Theorem 5.1. For $h$ sufficiently small,

$$
\begin{aligned}
& \left|u_{h}\right|_{\Gamma_{\text {out }}(\Omega)}^{2}+h^{3 / 2}\left|\left(u_{h}\right)_{\tau}\right|_{\Gamma_{\text {out }}(\Omega)}^{2}+\sqrt{h}\left\|\left(u_{h}\right)_{\alpha}\right\|_{\Omega}^{2}+\sigma\left\|\left(u_{h}\right)_{x}\right\|_{\Omega}^{2} \\
& \quad \leq C\left(\left|u_{h}\right|_{\Gamma_{\text {in }}(\Omega)}^{2}+h^{3 / 2}\left|\left(u_{h}\right)_{\tau}\right|_{\Gamma_{\text {in }}(\Omega)}^{2}+h^{-1 / 2}\left\|P_{n-2} f\right\|_{\Omega}^{2}+h^{1 / 2}\|f\|_{\Omega}^{2}\right) .
\end{aligned}
$$

Applying this result to $e_{h}=u_{h}-u_{I}$, and using the bounds in (4.11), we obtain

$$
\begin{aligned}
& \left|e_{h}\right|_{\Gamma_{\text {out }}(\Omega)}^{2}+h^{3 / 2}\left|\left(e_{h}\right)_{\tau}\right|_{\Gamma_{\text {out }}(\Omega)}^{2}+\sqrt{h}\left\|\left(e_{h}\right)_{, r}\right\|_{\Omega}^{2}+\sigma\left\|\left(e_{h}\right)_{x}\right\|_{\Omega}^{2} \\
& \leq C\left(1+\frac{\sigma^{2}}{h^{3}}\right) h^{2 n+1 / 2}\|u\|_{n+1, \Omega}^{2} .
\end{aligned}
$$

This leads to the following estimates:

Corollary 5.1. Assuming $\sigma \leq O\left(h^{3 / 2}\right)$ as $h \rightarrow 0$,

$$
\begin{aligned}
\left\|u-u_{h}\right\|_{\Omega} & \leq C h^{n+1 / 4}\|u\|_{n+1, \Omega}, \\
\left\|\left(u-u_{h}\right)_{\alpha}\right\|_{\Omega} & \leq C h^{n}\|u\|_{n+1, \Omega},
\end{aligned}
$$


and, applying (5.4) to the bounds on $\left(e_{h}\right)_{\tau}$ and $\left(e_{h}\right)_{\alpha}$ furnished by (5.11),

$$
\left\|\left(u-u_{h}\right)_{x}\right\|_{\Omega} \leq C h^{n-1 / 2}\|u\|_{n+1, \Omega}
$$

These bounds were shown in [1] to apply when $\sigma=0$. We have thus analyzed the finite element method (1.5) over the complete range $\sigma \geq 0$. A summary appears in Table 5.1.

TABLE 5.1. Error estimates for $\sigma=h^{p}$

\begin{tabular}{c|c|c|c|c} 
& $k$ & $\left\|u-u_{h}\right\|_{\Omega}$ & $\left\|\left(u-u_{h}\right)_{x}\right\| \Omega$ & $\left\|\left(u-u_{h}\right)_{\alpha}\right\|_{\Omega}$ \\
\hline$p \in[0,1]$ & $O\left(h^{2-p}\right)$ & $O\left(h^{n+p / 2}\right)$ & $O\left(h^{n}\right)$ & $O\left(h^{n-1+p}\right)$ \\
$p \in[1,1.5]$ & $O(h)$ & $O\left(h^{n+1-p / 2}\right)$ & $O\left(h^{n+1-p}\right)$ & $O\left(h^{n}\right)$ \\
$p \in[1.5, \infty)$ & $O(h)$ & $O\left(h^{n+1 / 4}\right)$ & $O\left(h^{n-1 / 2}\right)$ & $O\left(h^{n}\right)$
\end{tabular}

\section{BOUNDARY CONDITIONS}

We now consider the model problem (1.1) in a bounded domain $\Omega=[0,1] \times$ $[0, T]$ with $u$ given at $x=0$ and $x=1$. We take $\alpha_{1} \geq 0$, making the flow direction be left to right, and refer to triangles having a side along $x=0$ $(x=1)$ as inflow (outflow) triangles. We retain assumptions $\mathrm{H} 1-\mathrm{H} 4$ on the triangulation, with the possible exception of $\mathrm{H} 3$ along $x=0$ and $x=1$ as noted below.

Case A. $\sigma>O(h)$ as $h \rightarrow 0$. Here the diffusion term is dominant, and we must take $k<O(h)$ as $h \rightarrow 0$ for stability. Since H3 holds for triangle sides not lying along $x=0$ or $x=1$, all such sides must approach horizontal as $h \rightarrow 0$, implying that inflow triangles asymptotically are of type II and outflow triangles of type I. We assume this is invariably the case.

Inflow triangles require special consideration because $\mathrm{H} 3$ cannot be satisfied along the $x=0$ side as $h \rightarrow 0$. We can deal with this by relabelling the sides so that $\Gamma_{3}(T)$ is the $x=0$ side, rather than the outflow side as depicted in Figure 3.1. This changes $v_{h}^{*}$ in such a way that Lemma 3.3 and all subsequent results remain valid. Minor modifications are required in the proof of Lemma 3.5 to reflect the fact that the length of $\Gamma_{3}(T)$ is now $O(k)$ rather than $O(h)$, and $\theta_{1}, \theta_{2} \rightarrow \frac{\pi}{2}$ as $h \rightarrow 0$.

For an outflow triangle $T$, the inner product conditions (1.5) must be modified to accommodate the outflow boundary condition. We consider the most obvious alternative: fixing $u_{h}$ on the $x=1$ outflow side and treating the triangle as though it were of type II, with $\mathbf{P}_{n-2}(T)$ as the test space in (1.5). To deal with the fact that $\mathrm{H} 3$ will not be satisfied on $x=1$, we relabel the triangle sides so that $\Gamma_{3}(T)$ is the $x=1$ side. Lemmas 3.3 and 3.4 then still hold, and 
in place of Lemma 3.5, we have the following result:

Lemma 6.1. In an outflow triangle $T, u_{h}$ satisfies

$$
\begin{aligned}
& \oint \frac{\left(u_{h}\right)_{\tau}^{2}}{\alpha \cdot n} d \tau+\mu \frac{q}{\sigma}\left\|\left(u_{h}\right)_{\alpha}\right\|^{2} \\
& \leq C {\left[h^{-1}\left\|\left(u_{h}\right)_{x}\right\|^{2}+\left|\left(u_{h}\right)_{\tau}\right|_{\Gamma_{3}(T)}^{2}\right.} \\
&\left.\quad+\sigma^{-1}\left(q^{2}\left\|\left(u_{h}\right)_{\alpha}\right\|_{I(T)}^{2}+\left\|P_{n-2} f\right\|^{2}+q\|f\|^{2}\right)\right]
\end{aligned}
$$

for sufficiently small $q$, where $\mu$ is a positive constant.

Proof. Equations (3.11) and (3.14) are still valid, but (3.15) must be replaced by

$$
\begin{aligned}
\left|\frac{\sin \theta_{1} \sin \theta_{2}}{\prod_{i=1}^{3}\left(\alpha \cdot n_{i}\right)}\right|\left|\left(u_{h}\right)_{\alpha}\right|_{\Gamma_{3}(T)}^{2} & \leq C\left|\left(u_{h}\right)_{\alpha}\right|_{\Gamma_{3}(T)}^{2} \\
& \leq C\left(\left|\left(u_{h}\right)_{x}\right|_{\Gamma_{3}(T)}^{2}+\left|\left(u_{h}\right)_{\tau}\right|_{\Gamma_{3}(T)}^{2}\right) \\
& \leq C\left(h^{-1}\left\|\left(u_{h}\right)_{x}\right\|^{2}+\left|\left(u_{h}\right)_{\tau}\right|_{\Gamma_{3}(T)}^{2}\right) .
\end{aligned}
$$

The last inequality above reflects the fact that $\Gamma_{3}(T)$ has length $k$. Replacing $\sigma\left\|\left(u_{h}\right)_{x x}\right\|^{2}$ in (3.11) by $q \sigma\left\|\left(u_{h}\right)_{x x}\right\|^{2}$ where $q<1$, then using (3.14) and (6.2), we obtain the desired result.

We now multiply (6.1) by $\frac{M h^{2}}{q}$ and add to (3.3), as before. On the left side of (3.22), in place of $\xi \sigma\left\|\left(u_{h}\right)_{x}\right\|^{2}$, we now have $\sigma\left(\xi-\frac{C M h}{q \sigma}\right)\left\|\left(u_{h}\right)_{x}\right\|^{2}$. Since $\frac{h}{\sigma} \rightarrow 0$ as $h \rightarrow 0$, this term can be bounded by $\xi^{\prime} \sigma\left\|\left(u_{h}\right)_{x}\right\|^{2}$ for $h$ sufficiently small, where $\xi^{\prime}$ is a positive constant. The basic stability result of $\S 3$ and subsequent error estimates remain in effect.

Case B. $\sigma \leq O(h)$ as $h \rightarrow 0$. In this setting, the stability condition on $q=\frac{\sigma k}{h^{2}}$ permits us to take $k$ proportional to $h$ as $h \rightarrow 0$. Thus assumption $\mathrm{H} 3$ is inoperative. (In conjunction with the condition on $q, \mathrm{H} 3$ ensures compatibility between the domain of dependence requirements of a parabolic problem ( $\sigma>$ $O(h)$ ) and the explicit (hyperbolic) ordering of the triangles.) Inflow triangles are thus covered directly by the analysis of $\S \S 3$ and 4 .

If $\sigma$ is small compared to $h$, treating outflow triangles as in case A will result in oscillations. It is thus inappropriate for convection-dominated problems. Our strategy here will be to triangulate $\Omega$ in such a way that the outflow boundary is removed from the domain of dependence of $u_{h}$ in the interior. This could be achieved, for example, via the mesh shown in Figure 6.1, where the vertical lines act as barriers to right-to-left propagation of information. This triangulation is compatible with the conditions of our analysis, and the previous error estimates will be valid over a reduced domain which excludes those triangles that impinge on $x=1$. The effect of the boundary condition at $x=1$ will thus be confined to an outflow layer, where a special technique could be employed.

We note that the decay rate which separates cases $\mathrm{A}$ and $\mathrm{B}$ above also acts as a threshold for the streamline diffusion method [2,5], where for $\sigma<h$ 


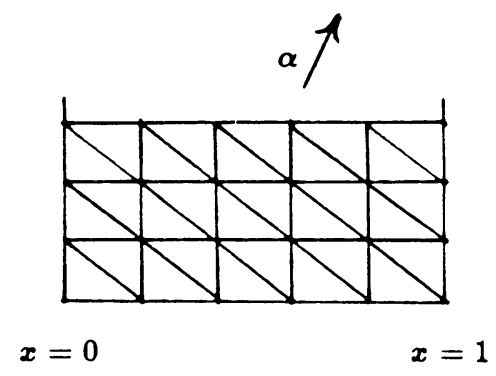

Figure 6.1

the Galerkin test functions are augmented by streamline derivatives to promote stability.

\section{Computational Results}

We present numerical results for the quadratic $(n=2)$ version of the finite element method, as applied to two test problems. The first problem,

$$
\begin{aligned}
& u_{\alpha}=\sigma u_{x x}, \quad x \in[0,2 \pi], \quad t \in(0,1), \\
& u(x, 0)=\sin x, \quad x \in[0,2 \pi], \\
& \text { periodic boundary conditions, }
\end{aligned}
$$

has solution

$$
u(x, t)=\sin \left(x-\frac{\alpha_{1}}{\alpha_{2}} t\right) \exp \left(-\frac{\sigma t}{\alpha_{2}}\right) .
$$

We take $\alpha$ to make an angle of 60 degrees with the horizontal and use a uniform isosceles triangulation of the domain, as depicted in Figure 7.1a. Along lines $t=$ constant, consecutive grid points for piecewise quadratic approximation are separated by $\Delta x=h / 2$. We take the "Courant number" $\frac{\alpha_{1} k}{\alpha_{2} \Delta x}$ to be less than 1 , so that $u_{h}$ is propagated upward through alternate layers of type I and type II triangles.

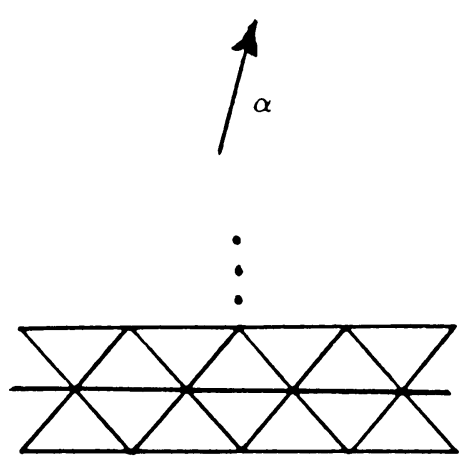

0
$2 \pi$

Figure $7.1 \mathrm{a}$

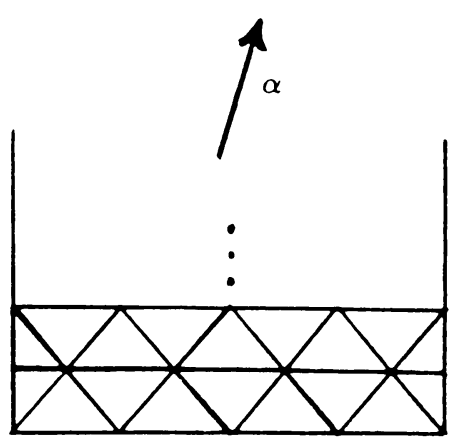

0

FIGURE $7.1 \mathrm{~b}$ 
The results in Table 7.1 correspond to $\sigma=1, \frac{\sigma k}{(\Delta x)^{2}}=.4$. The quantities shown are

$$
\max _{i} \sqrt{\int_{0}^{2 \pi}\left[u\left(x, t_{i}\right)-u_{h}\left(x, t_{i}\right)\right]^{2} d x},
$$

taken over horizontal grid lines $t=t_{i}$, and the analogous error in $\left(u_{h}\right)_{x}$. The number of grid points along such lines is $N=\frac{2 \pi}{\Delta x}$. Ratios of consecutive errors in both $u_{h}$ and $\left(u_{h}\right)_{x}$ have a limiting value of 4 , as predicted by our analysis. For this problem we experimentally observed a stability condition of $\frac{\sigma k}{(\Delta x)^{2}} \leq \frac{\alpha_{2}}{2}=.433$, the same as would occur for a finite difference discretization of (1.1) using a forward difference in time and centered differences in space.

TABLE 7.1

\begin{tabular}{rcccc}
\hline$N$ & error in $u_{h}$ & ratio & error in $\left(u_{h}\right)_{x}$ & ratio \\
\hline 8 & $.928(-1)$ & - & $.160(-1)$ & - \\
16 & $.194(-1)$ & 4.79 & $.509(-1)$ & 3.14 \\
32 & $.427(-2)$ & 4.54 & $.130(-1)$ & 3.90 \\
64 & $.992(-3)$ & 4.30 & $.276(-2)$ & 4.71 \\
128 & $.244(-3)$ & 4.06 & $.652(-3)$ & 4.24 \\
256 & $.608(-4)$ & 4.02 & $.160(-3)$ & 4.07 \\
\hline
\end{tabular}

Table 7.2 contains results for a convection-dominated case of (7.1) in which $\sigma=\Delta x$ and $\frac{\sigma k}{(\Delta x)^{2}}=.3$ (yielding Courant number .17). The ratios indicate that the optimal rate of convergence for quadratics, $O\left(h^{3}\right)$, is occurring, vs. a theoretical prediction of $O\left(h^{2.5}\right)$. We speculate that this improved rate is due to damping of the error as the approximate solution develops, as has been shown for the discontinuous Galerkin method for (1.2) [7].

TABLE 7.2

\begin{tabular}{rcc}
\hline$N$ & error in $u_{h}$ & ratio \\
\hline 8 & $.700(-1)$ & - \\
16 & $.139(-1)$ & 5.03 \\
32 & $.222(-2)$ & 6.27 \\
64 & $.296(-3)$ & 7.50 \\
128 & $.383(-4)$ & 7.74 \\
256 & $.486(-5)$ & 7.87 \\
512 & $.613(-6)$ & 7.93 \\
\hline
\end{tabular}

Table 7.3 provides justification for including the integral over $\Gamma_{\text {in }}^{*}(T)$ in (1.5). The results shown there are for the same conditions as in Table 7.2, except that the inflow integral was omitted in generating $u_{h}$. The order of accuracy degrades to $O\left(h^{2}\right)$. 
TABLE 7.3

\begin{tabular}{rcc}
\hline$N$ & error in $u_{h}$ & ratio \\
\hline 8 & $.475(-1)$ & - \\
16 & $.159(-1)$ & 2.98 \\
32 & $.487(-2)$ & 3.28 \\
64 & $.125(-3)$ & 3.90 \\
128 & $.318(-3)$ & 3.93 \\
256 & $.802(-4)$ & 3.96 \\
512 & $.201(-4)$ & 3.98 \\
\hline
\end{tabular}

The second test problem for which we give numerical results is

$$
\begin{aligned}
& u_{x x}=\sigma u_{x x}, \quad 0<x<1, t>0, \\
& u(x, 0)=0, \\
& u(0, t)=0, \quad u(1, t)=1, \quad t>0 .
\end{aligned}
$$

The steady state solution,

$$
u(x, \infty)=\frac{\exp \left(-\frac{\alpha_{1}}{\sigma}(1-x)\right)-\exp \left(\frac{-\alpha_{1}}{\sigma}\right)}{1-\exp \left(\frac{-\alpha_{1}}{\sigma}\right)},
$$

has a boundary layer at $x=1$ which becomes sharper as $\sigma \rightarrow 0$. Using the triangulation shown in Figure 7.1b, with boundary conditions provided for as in Case A of $\S 6$, we computed the numerical steady state solution and its $L^{2}$ error. The results shown in Table 7.4 correspond to $\alpha$ a .60 degree angle, as measured from the horizontal, $\sigma=.1$, and $\frac{\sigma k}{\Delta x^{2}}=.39$. These results are roughly consistent with an $O\left(h^{2}\right)$ rate of convergence.

TABLE 7.4

\begin{tabular}{rcc}
\hline$N$ & error in $u_{h}(x, \infty)$ & ratio \\
\hline 8 & $.140(-1)$ & - \\
16 & $.262(-2)$ & 5.34 \\
32 & $.651(-3)$ & 4.02 \\
64 & $.171(-3)$ & 3.80 \\
128 & $.444(-4)$ & 3.85 \\
\hline
\end{tabular}

Figure 7.3 compares grid values of $u_{h}(x, \infty)$ and $u(x, \infty)$ for $\sigma=10^{-4}$, $N=64$. The time step was chosen to yield Courant number .95 (making $\frac{\sigma k}{\Delta x^{2}}=$ $.0105)$. The main interest here is the minimal extent to which the boundary oscillation propagates back into the flow domain. However, if $\sigma$ is taken still smaller while keeping $h$ fixed, the oscillations become more severe, eventually causing $u_{h}$ not to reach a steady state. We can see that this must happen by taking $v_{h}=1$ in (1.5) (this is a valid test function for outflow triangles under 


\begin{tabular}{cccccccc}
\hline$u_{h}(x, \infty):$ & .000 & .000 & $\ldots$ & .000 & .001 & -.773 & 1.000 \\
$u(x, \infty):$ & .000 & .000 & $\ldots$ & .000 & .000 & .000 & 1.000 \\
\hline & $\quad$ & & & & & & $\mid$ \\
& $x=0$ & & & & & & $x=1$
\end{tabular}

FIGURE 7.3

the prescription of case A of $\S 6$ ) and summing over all triangles. For our test problem, with $\sigma=0$, this yields

$$
\int_{0}^{1} u_{h}(x, t) d x=-\frac{\alpha_{1}}{\alpha_{2}} t \rightarrow-\infty \quad \text { as } t \rightarrow \infty .
$$

A remedy would be to use the triangulation of Figure 6.1 . This would isolate the outflow boundary from the interior and yield $u_{h}=0$ as the steady state solution up to the next to last vertical line. The boundary layer could then be dealt with separately.

\section{ACKNOWLEDGMENT}

I thank the referee for a careful reading of the manuscript and many useful comments.

\section{BIBLIOGRAPHY}

1. R. S. Falk and G. R. Richter, Analysis of a continuous finite element method for hyperbolic equations, SIAM J. Numer. Anal. 24 (1987), 257-278.

2. C. Johnson, U. Nävert, and J. Pitkäranta, Finite element methods for linear hyperbolic problems, Comput. Methods Appl. Mech. Engrg. 45 (1984), 285-312.

3. C. Johnson and J. Pitkäranta, An analysis of the discontinuous Galerkin method for a scalar hyperbolic equation, Math. Comp. 46 (1986), 1-26.

4. P. Lesaint and P. A. Raviart, On a finite element method for solving the neutron transport equation, Mathematical Aspects of Finite Elements in Partial Differential Equations (C. deBoor, ed.), Academic Press, 1974, pp. 89-123.

5. U. Nävert, A finite element method for convection-difficion problems, Ph. D. thesis, Dept. of Computer Sciences, Chalmers Inst. of Tech., Göteborg, 1982.

6. W. H. Reed and T. R. Hill, Triangular mesh methods for the neutron transport equation, Los Alamos Scientific Laboratory Report LA-UR-73-479, 1973.

7. G. R. Richter, An optimal-order error estimate for the discontinuous Galerkin method, Math. Comp. 50 (1988), 75-88. 08903

Department of Computer Science, Rutgers University, New Brunswick, New Jersey 